\title{
Towards stable coupling methods for high-order discretization of fluid-structure interaction: Algorithms and observations
}

\author{
Robert M. Kirby ${ }^{\mathrm{a}, *}$, Zohar Yosibash ${ }^{\mathrm{b}}$, George Em Karniadakis ${ }^{\mathrm{c}}$ \\ ${ }^{a}$ School of Computing, University of Utah, 50 South Central Campus Dr., Room 3190, Salt Lake City, UT 84112, USA \\ b Pearlstone Center for Aeronautical Engineering Studies, Department of Mechanical Engineering, Ben-Gurion University, Beer-Sheva, Israel \\ ${ }^{\mathrm{c}}$ Division of Applied Mathematics, Brown University, Providence, RI, USA
}

Received 23 August 2005; received in revised form 11 August 2006; accepted 20 September 2006

Available online 2 November 2006

\begin{abstract}
Weak coupling of an explicit spectral/hp finite element ALE fluid solver with a Chebyshev collocation structural solver for fluid-structure interaction problems is addressed. When attempting to couple high-order spatial discretizations of both fluid and structural phenomena, we are required to consider first principles in order to answer two important questions: (1) What information (e.g. forces, velocities, displacements) does one transfer and when? and (2) How does one transfer information? Our goal is to address these questions by considering a non-conventional transfer of velocities of the wet-surface from the structure to the fluid, and the usual transfer of pressures on the wet-surface from the fluid to the structure. A subsonic three-dimensional compressible flow over an elastic non-linear plate model is considered as a representative example problem and we explore the various function spaces in which data (such as pressures, velocities and displacements) lie. We provide arguments as to what projection algorithms to use in combination with a time-staggering scheme to achieve stable and accurate results and at the same time to retain a temporal second-order scheme. We demonstrate the proposed algorithms by numerical examples considering long time integration.
\end{abstract}

(c) 2006 Elsevier Inc. All rights reserved.

Keywords: High-order methods; Fluid-structure interaction; Spectral/hp elements; Coupling algorithms; Data transfer

\section{Introduction}

Problems involving the coupled response of structures and flows have become of increasing interest in various disciplines such as aircraft structural components in flight (for example, [1-4]), marine risers in ocean currents (as discussed in [5]), and blood flow in arteries (for example [6] and reference therein). These problems are characterized by a dynamic and non-linear behavior both in the structure as well as fluid mechanics response, and impose a significant challenge from the numerical simulation viewpoint.

\footnotetext{
* Corresponding author. Tel.: +1 801585 3421; fax: +1 8015856513.

E-mail addresses: kirby@cs.utah.edu (R.M. Kirby), zohary@bgu.ac.il (Z. Yosibash), gk@cfm.brown.edu (G.E. Karniadakis).
} 
Recently, high-order methods (spectral or hp-finite elements (FEs)) have been shown to provide exponential convergence rates for both structure $[7,8]$ and fluid [9] simulations, thus a natural step is to investigate their performance in a coupled fluid-structure interaction scheme. These capabilities allow direct numerical simulations (DNS) of the flow to be combined with a fully three dimensional structural simulation of very thin structures without a priori modeling assumptions. To this end, we aim herein to investigate a weakly-coupled scheme which combines high-order (spectral and hp-FEMs) methods for discretizing both the fluid and structure. Because realistic flows are of interest, we are using a hp-discontinuous Galerkin CFD code in which an arbitrary-Lagrangian-Eulerian (ALE) formulation [10] is available. This choice of code necessitates transfer of velocities from the structural domain to the fluid domain on the mutual interface. Another important consideration is the wish to simulate relatively large deformations of a structure; therefore we consider the von-Kármán plate problem which is discretized in space with a Chebyshev-collocation spectral method. The difference between the spaces in which the solution is sought in the two solvers becomes of major importance when highorder methods are coupled, a problem which is studied in detail. Finally, use of high-order methods for spatial discretization suggests the use of high-order time step integration, which has to be stable, and that the coupled system retain the time-marching order of each its components - the structure and fluid solvers.

\subsection{Objectives}

There are four important issues that have to be addressed when coupling schemes are used in conjunction with high-order methods:

Conservation: The coupling of the fluid and structure is achieved by ensuring that tractions are applied by the fluid to the structure and that the structure reacts by either passing displacements or velocities which, in turn, deform the fluid mesh. As the numerical schemes which deform the mesh in the fluid solver (using the arbitrary Lagrangian-Eulerian (ALE) scheme [10]) obtain as input either displacements or velocities, only one of these quantities is, in general, preserved across the interface. Therefore, weakly-coupled schemes do not, in general, conserve all properties, but rather a subset of properties determined by the particular assumptions invoked in the development of the numerical schemes. We herein are restricted to pass velocities and thus elect to conserve: (a) total force and (b) total momentum at the interface. Since structural displacements are not directly transferred, but are computed by the fluid solver through a time integration approximation, the energy of external forces is not conserved; it serves as a measure for energy (or "error") control.

Projection: Determination of the proper projection of the tractions (herein we are interested in pressures only and ignore viscous effects between fluid and structure) applied on the wetted surface into the functional space spanned by the structural solver discretization, and the projection of the velocities of the wetted surface into the functional space spanned by the fluid solver discretization. An intrinsic property of a weaklycoupled system is the transfer of forces on the wetted surface from the fluid to the structure [11] and the transfer of velocities from the structure to the fluid. An improper transfer of this information may lead, in the best case, to deterioration of the temporal order of the coupled scheme, and in the worst case to a loss of stability. This loss of stability in high order methods does not always mean loss of energy conservation through the fluid-structure interface. As shall be discussed, when passing velocities from a higher order discretization (structure) to a lower order discretization (fluid), loss of stability may occur due to the excitement of energy due to aliasing, having nothing to do with the coupling scheme. This phenomenon is a feature highlighted by high-order methods and often invisible in low-order formulations due to their dissipative nature.

Staggering: Determination of the staggering algorithm, i.e. the instances at which information is transferred from one code to the other. Although both the wetted surface displacements and velocities are to be transferred from the structure to the fluid code at each instance, usually only displacements or velocities are transferred, and the other "field" is inferred from this information.

Convergence: Quantification of the rate of temporal convergence. Consider, for example, a fluid solver designed for solving moving domain problems having a temporal order of accuracy of $(\Delta t)^{n}$ and a structural 
solver of the same temporal order of accuracy: one would like to ensure that the coupled system maintains an order of accuracy $(\Delta t)^{n}$.

Herein we present the issues which arise in developing weakly-coupled high-order numerical algorithms for simulating FSI problems in which one is interested in the long time response of the system. To meet our objectives, we focus on two fundamental issues. First, we describe the necessary quantities and the precise transfer instances at which they are transferred for the two solvers of interest. Secondly, and of equal importance, we examine the function spaces formed by the different spatial discretization methods of the two solvers in an attempt to understand what requirements (e.g. conservation, monotonicity) should be placed upon the transfer methodology.

Algorithmic procedures and numerical evidence are provided addressing the transfer of pressures on the wetted surface from the fluid to the structure and velocities from the structure to the fluid. The ramifications of different choices in the coupling and projection methods on the stability of the system are demonstrated by numerical examples, and insights are provided to help practitioners in their efforts to couple high-order discretization methods in the modeling of FSI phenomena.

\subsection{Background}

Solution of the entire set of equations as one discretized system, referred to as the monolithic approach [1214], is attractive in that such systems are often amenable to mathematical analysis and hence allow construction of schemes which have desired properties such as conservation. However, the monolithic approach is often inefficient due to its attempt to capture with one discretization methodology the completely different spatial and temporal characteristics of the flow and the structure. The second approach often mentioned is the notion of strong-coupling, referring to solvers which might use different discretizations for the fluid and the structure but which employ sub-iterations in each time-step to enforce strong coupling between the fluid and the structure.

A more practical approach is referred to the loosely-coupled, weakly-coupled or staggered method [15]. In this case the governing equations for the fluid and structure are discretized separately in each of the subdomains and coupled using a synchronization procedure both in time and space without sub-iteration. Weakly-coupled schemes have been extensively applied to a variety of different fluid-structure-interaction (FSI) problems of engineering interest in the past ten years. However due to the complexity of both the fluid and structural non-linear equations, simplifying assumptions are often employed in either the fluid or the structural solver. On the part of the fluid solvers, modeling assumptions such as inviscid flow or viscous flow with turbulence modeling are used to simplify the fluid computation. On the part of the structure, linear elasticity is usually assumed, and thin solid structures are modeled with combinations of beam, plate and shell elements, each of which are formulated upon certain assumptions. As was pointed out in [16], these simplifications have led to advancement in the area of fluid-structure interaction, however the assumptions under which these simplifications are made lead to discretization as well as idealization (modeling) errors - errors which need to be quantified.

During this same period there has been significant progress in the development of high-order methods for solving fluid mechanics and solid mechanics problems. For instance, discontinuous Galerkin spectral/hp element methods [17] have been developed which enable direct numerical simulations (DNS) in complicated geometries on moving meshes. Concurrent development of spectral, p- and hp-finite element (FE) methods in solid mechanics have shown remarkable superior performance over low-order conventional FEM in elasticity and continuum mechanics applications [7,18], especially when slender domains are of interest. In an effort to control the discretization errors and minimize idealization errors, the use of an explicit parallel spectral/hp element DNS fluid solver based on discontinuous Galerkin formulation (enabling a good and fast resolution of the flow field) weakly-coupled to a high-order (pseudo-spectral) solver of the solid mechanics problem, is advocated. To this end, the coupling algorithm for the two high-order methods is a crucial component which has not yet been systematically investigated.

The starting point for most coupling investigations is a discussion of the temporal discretization applied both in the flow and in the structure. In $[1,19,20]$, an implicit strong-coupling algorithm for time marching 
is applied, thus an iterative approach using the Newton-like sub-iteration is adopted in which pressure is transferred from the fluid to the structure, and displacements are transferred back from the structure to the fluid after each sub-iteration. This eliminates errors from linearization and factorization as well as from lagged boundary conditions. In [1] the effect of instability is demonstrated for lagged fluid-structure coupling, and is removed via the sub-iteration approach. As an alternative to sub-iteration, staggered weakly-coupled algorithms which employ prediction-correction as part of the temporal coupling of passing forces from the fluid to the structure and displacements from the structure to the fluid have been advocated [2,21-23] and shown to be second-order accurate in time [24].

All of the previously mentioned FSI examples have coupled relatively low-order fluid discretization with low-order structural discretization. Herein we aim at systematically investigating the weak-coupling of a high-order fluid solver to a high-order non-linear structural solver. Specifically, we attempt to answer two fundamental questions: (1) What does one transfer and when? and (2) How should the data be transferred? Upon attempting to answer these questions for high-order methods, we are able to elucidate the advantages and trade-offs of various coupling and projection schemes.

As our driving example problem, we have chosen to perform a direct numerical simulation (DNS) of threedimensional flow over a plate using an explicit discontinuous Galerkin spectral/hp element fluid solver [10] coupled to a high-order structural solver simulating a von-Kármán non-linear model of a plate [25]. The differences in spatial and temporal discretizations of these two solvers is characteristic of the types of high-order fluid and structural solvers we are interested in coupling, and hence they provide us a rich testing environment in which to explore answers to our two fundamental questions.

One prevalent characteristic of high-order methods is that their successful application often requires one to address first principles. High-order methods are, in general, more susceptible to numerical instabilities than other low-order discretizations. This could be frustrating for the users who seek robustness, but it is actually safe-guarding against erroneous answers, as typically high-order codes blow up in seriously under-resolved simulations. In under-resolved high-order discretizations there are many more wiggles, and therefore it is easier to detect suspicious simulations before resorting to more rigorous error estimation. Also, high-order discretizations are typically associated with little numerical dissipation unlike finite-difference (or low-order finite volume) methods, which introduce an erroneous numerical dissipation in low resolution discretizations. The numerical methods literature is replete with examples in which assumptions as to the low-order nature of the discretization are firmly embedded into the methodology, sometimes to the point that computational scientists do not realize the employment of such assumptions (see vivid examples in [26,27]).

\subsection{Outline}

The paper is organized as follows. We provide the notations and mathematical formulation of the fluid and structural problems in Section 2. In Section 3 the numerical schemes for spatial and temporal discretization are reviewed for each of the solvers separately. The two fundamental questions when coupling two discretizations: (1) "What information does one transfer and when?" and (2) "How does one transfer information?" are addressed in Sections 4 and 5, respectively. Numerical results are presented and discussed in Section 6. We conclude in Section 7 with a summary of our findings, conclusions and future work.

\section{Problem formulation}

Herein the FSI problem is mathematically described, and a common notation is introduced in an attempt to make the description accessible to both the fluid mechanics and solid mechanics communities. We denote by $\Omega(t) \in \mathbb{R}^{3}$ the entire three dimensional domain of interest, which has two distinct subdomains $\Omega_{\mathrm{F}}(t)$ and $\Omega_{\mathrm{S}}(t)$ such that $\Omega=\Omega_{\mathrm{F}}(t) \cup \Omega_{\mathrm{S}}(t)$. The subdomain $\Omega_{\mathrm{F}}(t)$ denotes the region on which the equations of motion for the fluid are solved; the subdomain $\Omega_{\mathrm{S}}(t)$ denotes the deformed configuration of the structure given by the structure's equations of motion. The fluid-structure interface, also called the "wetted surface," is denoted by $\Gamma_{\mathrm{FS}}(t) \stackrel{\text { def }}{=} \Omega_{\mathrm{F}}(t) \cap \Omega_{\mathrm{S}}(t)$.

As a model problem we consider a 3-D viscous subsonic compressible flow over a dynamic von-Kármán non-linear square plate embedded in a rigid wall as shown in Fig. 1. 


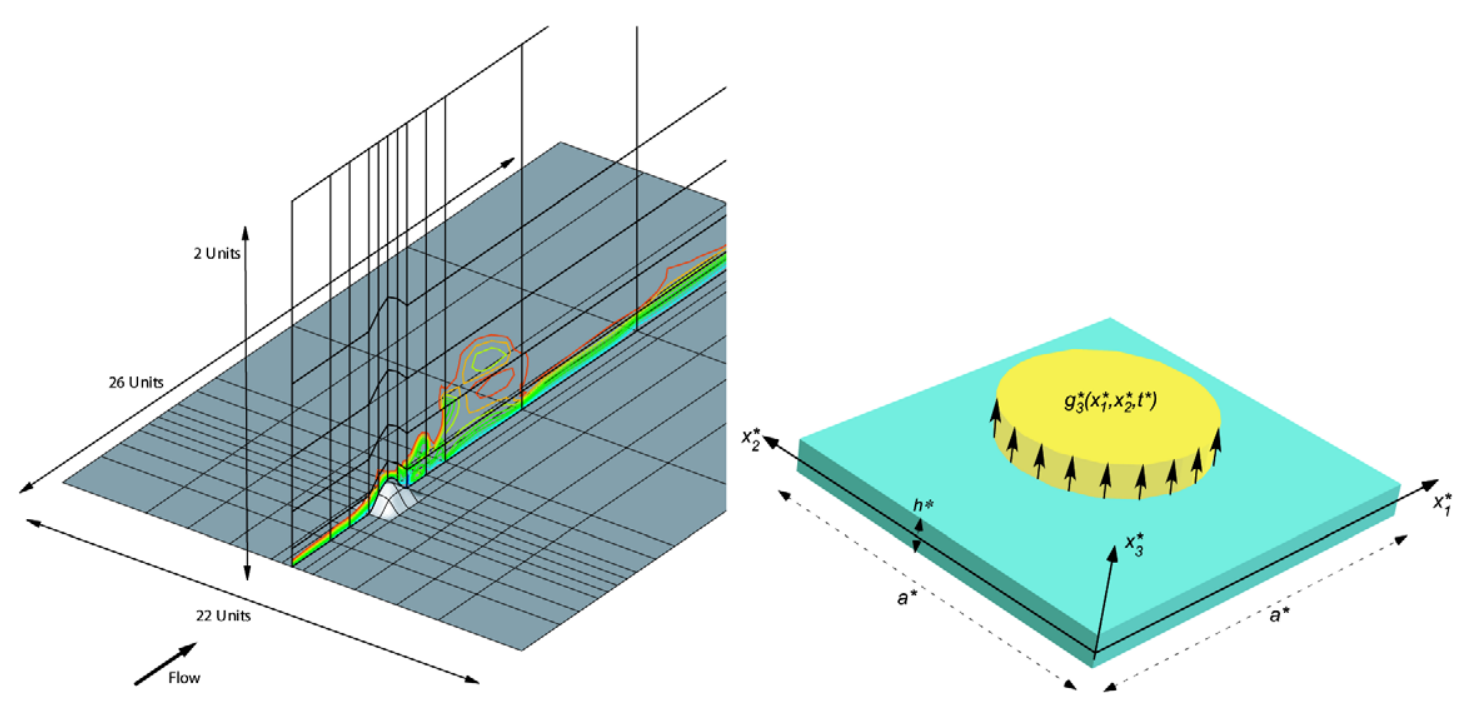

Fig. 1. The FSI model problem under consideration. Left: We present the computational fluid domain $\Omega_{\mathrm{F}}(t)$ (specified in non-dimensional units) where the solid black lines give an indication of the mesh used (edges of hexahedra elements). The deflected plate (representing the wetted surface $\left.\Gamma_{\mathrm{FS}}(t)\right)$ and corresponding mesh displacement above the plate is shown by the cut-plane presented. Contours of streamwise velocity are shown to indicate the affect of the deflected plate on the fluid solution. Right: We present the computational structural domain $\Omega_{\mathrm{S}}(t)$ with notation used in the text.

An overview of the governing equations used to model the fluid and structure is provided in the sequel. In particular, we focus on two critical issues: the compatibility conditions which must exist between the fluid domain and the structural deformed configuration, and the non-dimensionalization compatibility which must be maintained when passing field information.

\subsection{The governing equations of the FSI system}

The fluid and the structure are dictated by two different sets of governing questions with matching boundary conditions on the interface $\Gamma_{\mathrm{FS}}(t)$. The governing equations are expressed by non-dimensional quantities, so to distinguish between the physical and non-dimensional quantities we denote the physical values by an asterisk. A compact form of the FSI system can be expressed as follows:

$$
\begin{aligned}
& \dot{\boldsymbol{W}}+\nabla \cdot \mathbf{F}\left(\boldsymbol{W}, \boldsymbol{U}^{g}\right)=\frac{1}{R e_{\infty}} \nabla \cdot \mathbf{F}^{v}(\boldsymbol{W}) \quad \text { on } \Omega_{\mathrm{F}}(t) \\
& m \ddot{\boldsymbol{u}}+c \dot{\boldsymbol{u}}+k(\boldsymbol{u})=\mathbf{g} \quad \text { on } \Omega_{\mathrm{S}}(t)
\end{aligned}
$$

where Eq. (1) denotes the compressible Navier-Stokes equations written in arbitrary Lagrangian-Eulerian (ALE) form (see Appendix A) and Eq. (2) denotes the non-linear von-Kármán equations, where $k(\boldsymbol{u})$ is a nonlinear function of the displacements (see Appendix B). With respect to the governing equations for the fluid given by (1), the vector $\boldsymbol{W}^{\mathrm{T}}(\boldsymbol{x}, t)=\left(W_{1}, W_{1} W_{2}, W_{1} W_{3}, W_{1} W_{4}, W_{5}\right)^{\mathrm{T}}, \boldsymbol{x} \in \Omega_{\mathrm{F}}(t)$, denotes the fluid solution variables of density, momentum in the three coordinate directions with fluid velocity $\left(W_{2}, W_{3}, W_{4}\right)^{\mathrm{T}}$, and total energy, respectively, the vector $\boldsymbol{U}^{g}$ denotes the velocity of the moving frame within the ALE formulation, $R e_{\infty}$ denotes the Reynolds number, and $\mathbf{F}$ and $\mathbf{F}^{v}$ denote that inviscid and viscous fluxes respectively. With respect to governing equations of the structure given by (2), the vector $\boldsymbol{u}^{\mathrm{T}}(\boldsymbol{x}, t)=(u, v, w)^{\mathrm{T}}, \boldsymbol{x} \in \Omega_{\mathrm{S}}(t=0)$ denotes the displacement of the structure in the three coordinate directions, $m, c, k(\boldsymbol{u})$ denote the mass, internal dissipation and non-linear stiffness of the structure, respectively, and $\mathbf{g}$ denote forces (tractions and integrated body forces in the thickness direction) on the structure, respectively.

Details of the compressible Navier-Stokes ALE formulation and the von-Kármán model used in this work can be found in $[9,10,25]$. The salient points which need to be mentioned for the current discussion are the following: 
- The loading on the structure by the fluid (i.e. pressure and viscous forces) can be computed from the fluid state vector $\boldsymbol{W}$.

- The displacement $\boldsymbol{u}$ of the structure denotes the position of the deformed configuration $\Omega_{\mathrm{S}}(t)$, and correspondingly $\dot{\boldsymbol{u}}$ denotes the velocity of the structure. This information dictates the deformation of the fluid domain $\Omega_{\mathrm{F}}(t)$ and corresponding frame velocity $\boldsymbol{U}^{g}$ used in the ALE formulation.

- The non-dimensionalizations used for the fluid equations and structural equations are typically different. For instance, the fluid is often non-dimensionalized through the specification of a characteristic length $a^{*}$, pressure $p_{\infty}^{*}$, fluid density $\rho_{\infty}^{*}$, dynamic viscosity $\mu_{\infty}^{*}$ and thermal conductivity $\kappa_{\infty}^{*}$, from which nondimensional parameters such as the Reynolds number $R e_{\infty}$ and Mach number $M a_{\infty}$ can be derived. The structure, on the other hand, is normally non-dimensionalized based upon a characteristic length $a^{*}$, thickness $h^{*}$, Young modulus $E^{*}$, Poisson ratio $v$, structural viscosity coefficient $c^{*}$ and structural density $\rho_{\mathrm{S}}^{*}$. Because of these difference in non-dimensionalization, it is necessary to convert quantities passed between the two systems. A unified non-dimensionalization is presented in Appendix C.

\subsection{The interface $\Gamma_{F S}(t)$}

Because there are two separate domains with different sets of equations, the coupling is realized through the wetted surface which is the common boundary $\Gamma_{\mathrm{FS}}(t)$ (i.e. its shape and boundary conditions). It is important to realize that in the weak coupling strategy proposed herein, $\Gamma_{\mathrm{FS}}(t)$ has different geometrical realizations when viewed in the fluid or the structural solvers, because different discretizations are used to represent the interface on the fluid and structure and because velocities are transferred from the structure to the fluid solver, unlike displacements in other common coupling schemes. Thus, we denote by $\Gamma_{\mathrm{FS}}^{\mathrm{F}}(t)$ the interface as realized in the fluid solver, and by $\Gamma_{\mathrm{FS}}^{\mathrm{S}}(t)$ the one realized in the structural solver. The geometrical description of $\Gamma_{\mathrm{FS}}^{\mathrm{F}}(t)$ is dictated by the velocities $\left.\dot{\boldsymbol{u}}(\boldsymbol{x}, t)\right|_{\Gamma_{\mathrm{FS}}^{\mathrm{S}}}$, the trace of structural velocities field on $\Gamma_{\mathrm{FS}}^{\mathrm{S}}(t)$. The forces that the fluid apply on $\Gamma_{\mathrm{FS}}^{\mathrm{S}}(t)$ are the forces $g_{i}$ applied to Eq. (2), computed on $\Gamma_{\mathrm{FS}}^{\mathrm{F}}(t)$.

Denoting the set of points on the fluid-structure interface at $t=0$ by $\boldsymbol{x}_{\mathrm{FS} 0} \in \Gamma_{\mathrm{FS}}(0)$, then mathematically, these conditions are given by:

$$
\begin{aligned}
& \Gamma_{\mathrm{FS}}^{\mathrm{S}}(t)=\left\{\boldsymbol{x} \mid \boldsymbol{x}=\boldsymbol{x}_{\mathrm{FS} 0}+\operatorname{trace}(\boldsymbol{u}(\boldsymbol{x}, t))\right\} \\
& \Gamma_{\mathrm{FS}}^{\mathrm{F}}(t)=\left\{\boldsymbol{x} \mid \boldsymbol{x}=\boldsymbol{x}_{\mathrm{FS} 0}+\operatorname{Int}[\operatorname{trace}(\dot{\boldsymbol{u}}(\boldsymbol{x}, t))]\right\} \\
& -p=g_{3}, \quad \text { on } \Gamma_{\mathrm{FS}}^{\mathrm{S}}(t) \\
& \left(\sigma_{i j}\right)_{\mathrm{F}} n_{j}=g_{i}, \quad i=1,2 \quad \text { on } \Gamma_{\mathrm{FS}}^{\mathrm{S}}(t) \\
& \dot{\boldsymbol{u}}(\boldsymbol{x}, t)=\frac{\partial \boldsymbol{u}}{\partial t}=\left(W_{2}, W_{3}, W_{4}\right)^{\mathrm{T}} \quad \text { on } \Gamma_{\mathrm{FS}}^{\mathrm{F}}(t)
\end{aligned}
$$

Eq. (4), states that the position of the interface in the fluid domain $\Omega_{\mathrm{F}}(t)$ is computed by the integrated velocities transferred from the structure and the deformed configuration $\Omega_{\mathrm{S}}(t)$ is given by the displacements of the structure from the reference position $\Omega_{\mathrm{S}}(t=0)$ Eq. (3). Eq. (5) requires the continuity of the pressure across the interface (because the displacements are very small, the pressure is applied as acting in the $x_{3}$ direction, i.e. is equal to $g_{3}$ ). Eq. (6) requires the shear stress to be continuous across the interface. Because the shear stress is negligible in this study compared to the pressure, we assume for simplicity that $g_{1}=g_{2}=0$. The fourth equation, given by (7) states that the fluid velocity at the interface should be equal to the velocity of the structure (i.e. no slip and no penetration).

As will be discussed, in a weakly-coupled collocated scheme, the displacements and the velocities of the fluid-structure interface realized by the structure and the fluid domains cannot be identical. Therefore, we apply a scheme which satisfies Eq. (7) at every integration time step.

\section{Solvers}

In this section we discuss the two solvers used in this study, namely the $\mathscr{N} \varepsilon \kappa \mathscr{T} \alpha r$ fluid flow solver and the von-Kármán structural solver. We emphasize those properties of the solvers necessary for discussing the temporal and spatial coupling strategies presented in Sections 4 and 5. 


\subsection{Fluid solver $-\mathscr{N} \varepsilon \kappa \mathscr{T} \alpha r$}

The flow solver used in this study corresponds to a particular version of the explicit discontinuous Galerkin spectral/hp element simulation code $\mathscr{N} \varepsilon \kappa \mathscr{T} \alpha r$, which is a general purpose CFD code for simulating incompressible, compressible and plasma flows in unsteady two-dimensional and three-dimensional geometries. The algorithmic details are discussed in $[9,28]$. The code uses meshes similar to standard finite element and finite volume meshes, consisting of structured or unstructured grids or a combination of both. Spectral/hp element discretizations provide resolution fidelity through a combination of elemental discretization (h-refinement) and polynomial expansion order per element (p-refinement). The particular variant of $\mathscr{N} \varepsilon \kappa \mathscr{T} \alpha r$ used in this study corresponds to the three-dimensional moving domain (ALE) compressible Navier-Stokes version presented in [10]. Note that:

- The code provides first, second- or third-order time integration on moving mesh problems. The explicit Adams-Bashforth schemes are used for time integration of both the fluid equations of motion and the mesh displacement (including the displacement of the wetted surface). The ALE time stepping implementation respects the discrete geometric conservation laws [29-31] automatically by solving the fluid equations of motion in the spatial configuration $\Omega_{\mathrm{F}}(t)$. For all results presented in this paper, second-order explicit time integration was used.

- The discontinuous Galerkin method is used for discretization of the fluid equations. Hence, fluid quantities are expressed as piecewise-discontinuous polynomial solutions. In the context of this work, the important observation is that the pressure on the wetted surface from the view of the fluid solver is a piecewise-discontinuous polynomial function residing in the function space $\mathscr{S}_{1}$ in Fig. 5.

- Although piecewise-discontinuous polynomial functions are admissible for the fluid variables, mesh displacements and velocities are required to remain $C^{0}$ continuous. This additional constraint on the mesh displacement/velocity is fully consistent with the DG formulation since the $C^{0}$ polynomial space for the wetted surface under consideration is merely a subspace of the $L^{2}$ piecewise-continuous polynomial space (as shown in Fig. 5). In the context of this work, note that the velocity on the wetted surface from the view of the fluid solver is a piecewise-continuous polynomial function (i.e. all velocity projection methods investigated in this paper project the structural velocity to piecewise-continuous polynomial functions).

\subsection{Structural solver - von-Kármán}

The non-dimensional form of the simplified von-Kármán plate model is solved numerically using a pseudo-spectral Chebyshev-collocation method for the spatial discretization and the Newmark- $\beta$ scheme for integration in time. Due to the bi-harmonic operator, a finite-element formulation requires $C^{1}$ continuous elements, thus considerably affects the computational complexity. The Chebyshev-collocation method, on the other hand, can easily treat the non-linear terms and is a natural choice for quadrilateral domains, and thus has been adopted in the present work. In all computations presented henceforth we use a grid of $(N+1) \times(N+1)$ points representing a tensor product interpolating polynomials of order $N$ in each direction based upon the Chebyshev-Lobatto points. Thus, each of the unknown functions, $u, v, w$ is approximated by a polynomial of the form $\mathscr{P}^{N}\left(x_{1}\right) \mathscr{P}^{N}\left(x_{2}\right)$. Clamped boundary conditions are enforced strongly on the approximating polynomial space. A detailed analysis of the numerical methods and their convergence properties for the full and simplified von-Kármán models is provided in [25,32], where we have shown that the chosen method is very efficient for spatial-discretization, yielding "exponential (spectral) converge rates".

The average acceleration variant of the Newmark- $\beta$ scheme (with Newmark parameters $\gamma=\frac{1}{2}$ and $\beta=\frac{1}{4}$ ) which exhibits second-order convergence in time and is unconditionally stable under linear analysis was chosen for time marching. The Newmark scheme requires the implicit evaluation of both the linear and non-linear spatial terms, where all the non-linear terms and forcing terms are grouped into the expression $G(\boldsymbol{u})$. Denoting by the subscript $n$ the quantities at time step $t_{n}$, then $\boldsymbol{u}_{n+1}$ is computed by the implicit Newmark scheme: 


$$
\left(\frac{4}{(\Delta t)^{2}}+\frac{2 c}{\Delta t}\right) \boldsymbol{u}_{n+1}=G\left(\boldsymbol{u}_{n+1}\right)+\left(\frac{4}{(\Delta t)^{2}} \boldsymbol{u}_{n}+\frac{4}{\Delta t} \dot{\boldsymbol{u}}_{n}+\ddot{\boldsymbol{u}}_{n}\right)+c\left(\frac{2}{\Delta t} \boldsymbol{u}_{n}+\dot{\boldsymbol{u}}_{n}\right) .
$$

Remark 1. It is important to notice that for the Newmark scheme used by the von-Kármán structure solver the load on the plate has to be available at the end of the time-step, i.e. at $t_{n+1}$. As will be discussed in detail in the following section, this condition is satisfied because the coupling strategy provides the pressure from $\mathscr{N}_{\varepsilon \kappa} \mathscr{T} \alpha r$ after being computed at $t_{n+1}$.

A fixed-point iteration with tolerance of $10^{-10}$ was used to accomplish the necessary implicit solve due to the non-linearities of the von-Kármán system (for further details see [25]).

\section{Temporal coupling strategy: what to transfer and when}

In this section we discuss the particular temporal coupling strategy we have adopted for coupling the two particular solvers mentioned in the previous section. In our discussion, we assume that the shear stresses that the fluid apply on the plate are negligible compared to the pressure (normal stress to the surface), and that the in-plane displacements/velocities of the plate are negligible compared to the deflection $w$ and transversal velocity $\dot{w}$. Thus, our discussion focuses on when should pressure be passed from the fluid to the structure, and when should the transversal velocity $\dot{w}$ of the plate be passed from the structure to the fluid.

\subsection{Weakly-coupled staggering strategy}

A multitude of different weakly-coupled schemes exist in the literature (e.g. [2,4,21,23]), and as pointed out in [15], even the same scheme seems to take on different monickers. The coupling literature is full of schemes mentioned as "staggered" or "segregated" or "time-lagged" algorithms. Each of these has its different merits, and the utility of a particular scheme is sometimes highly dependent upon the underlying time discretizations used in the fluid and structural solvers [24].

Because our fluid solver uses fully explicit time-stepping in both the fluid variables and in the mesh quantities, we have elected to use a modified version of the so-called "simple staggered algorithm" [2] as presented in Fig. 2. The time-stepping scenario expressed in explicit parts for advancing the entire FSI system from time level $n$ to time level $(n+1)$ is as follows:

- Part 1: At time $t_{n}$ the $\mathscr{N} \varepsilon \kappa \mathscr{T} \alpha r$ solver is explicitly advanced by a $\Delta t$ chosen so that the stability condition (e.g. CFL condition) is satisfied. Within this step, both the fluid variables $\boldsymbol{W}$ and the computational fluid domain $\Omega_{\mathrm{F}}(t)$ (i.e. mesh position in the ALE framework) are updated based upon current information (at $t_{n}$ ) and previous information (at $t_{n-j}, j=1, \ldots, r$ where $r$ is dictated by the Adams-Bashforth scheme used). The details of how this is accomplished are found in [10].

- Part 2: The pressure on the wetted surface at time level $n+1$ is "passed" using some projection algorithm (discussed in more detail in Section 5) to the structural solver.

- Part 3: Using the new load on the structure given at time level $(n+1)$, the structural solver using the Newmark scheme can advance the structural variables (displacements and velocities) to time level $(n+1)$.

- Part 4: The velocity of the interface at time level $(n+1)$ as computed by the structural solver is "passed" (projected) to be used by the fluid solver for updating the ALE mesh velocity $\mathbf{U}^{g}$ and for updating the mesh and wetted interface at the next time level.

Remark 2. In the above scheme, the time stepping algorithm used in the fluid and the time stepping algorithm used in the structure are independently second-order. As has been pointed out in [24], this does not necessarily imply that the coupled system will be second-order. Because the fluid solver is advanced in a fully explicit manner and because the Newmark scheme, which requires loads at time level $n+1$, is used for the structure, 


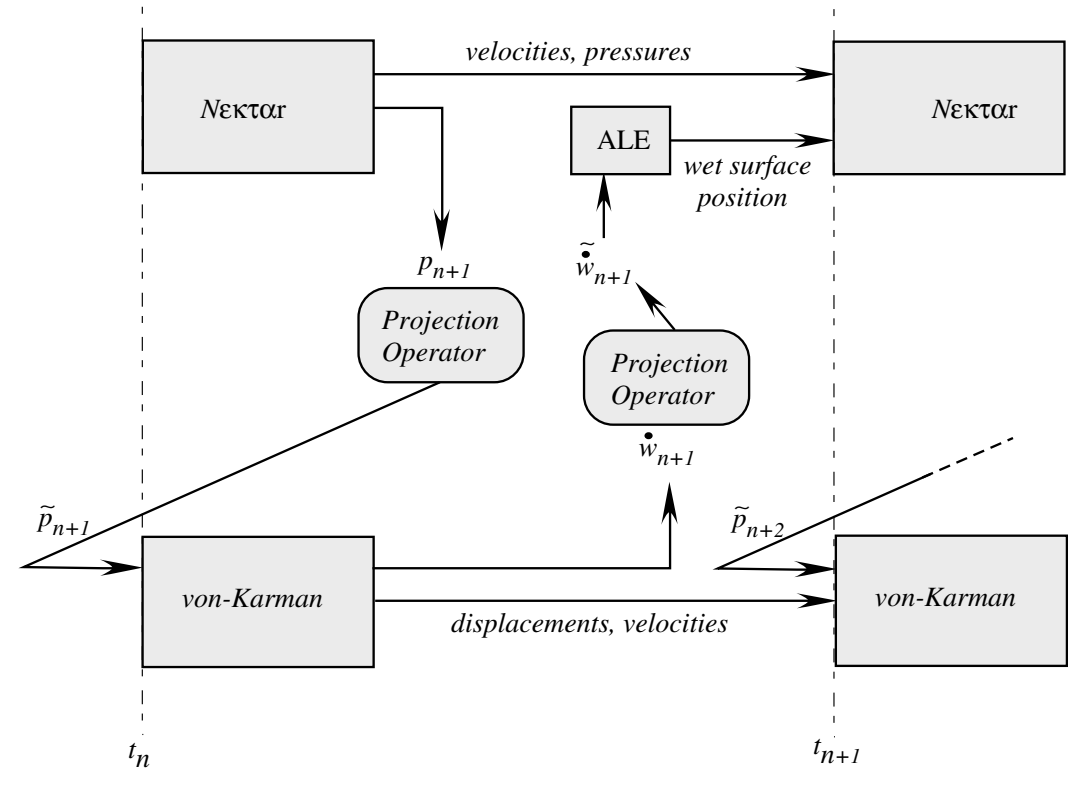

Fig. 2. Schematic representation of the staggered algorithm.

the algorithm just presented numerically demonstrates second-order accuracy of the coupled system. This is because the particular scheme above is in form part of the second-order IMEX-BDF (explicit-implicit) family of schemes [33], also see numerical demonstrations in [10].

Remark 3. As opposed to many coupled systems in which displacements are passed from the structure to the fluid and hence velocity of the structure must be inferred for use within the ALE framework, we have chosen to pass velocity and infer displacement as this is how the $\mathscr{N} \varepsilon \kappa \mathscr{T} \alpha r$ ALE solver [10] was designed. There are, of course, different ramifications depending on which option one chooses. If one passes displacements (as is commonly done), the position of the wetted surface with respect to the fluid solver and with respect to the structural solver will be identical (assuming discretization spaces within the fluid and solid which allow it; if the two function spaces representing displacement within the fluid and structural solvers do not intersect, then "identical" is impossible - we will comment on this further in Section 5). However, since in most collocated schemes of this form the velocity of the interface is inferred (normally to satisfy the discrete GCL [29,30]) there is a mismatch of momentum at the interface [23]. By passing velocities, we can develop algorithms which enforce a conservation (in space) of total momentum over the interface at the cost of the position of the wetted interface being slightly different. Further details will be provided in Sections 4.2 and 5.

Remark 4. In our current implementation, we do not implement sub-cycling - that is, we do not advance one solver through many small cycles holding the other solver fixed. This technique is common due to the difference in time scales between the fluid and the structure.

\subsection{Simple demonstrative coupling example}

To help demonstrate the IMEX-BDF (explicit-implicit) nature of the coupling scheme we use, we have generated a simple contrived example of coupled first-order ODE (that mimics $\mathscr{N} \varepsilon \kappa \mathscr{T} \alpha r$ time-stepping algorithm) and a second-order ODE (that mimics von-Kármán time-stepping algorithm) upon which numerical tests with exact solutions can be accomplished.

Consider the Adams-Bashforth time-marching scheme (used in $\mathscr{N} \varepsilon \kappa \mathscr{T}$ ar solver) with the Newmark scheme (used in von-Kármán solver) used to solve the following system: 


$$
\begin{aligned}
& \dot{\eta}_{1}=-\omega \eta_{1}-\omega \eta_{2}+\dot{\eta}_{2} \\
& \ddot{\eta}_{2}=-\omega \eta_{1}-\omega^{2} \eta_{2}+\dot{\eta}_{2}
\end{aligned}
$$

and let us investigate solutions of the form: $\eta_{1}(t)=\cos (\omega t), \eta_{2}(t)=\sin (\omega t)$ with initial conditions $\eta_{1}(0)=1$, $\eta_{2}(0)=0$ and $\dot{\eta}_{2}=\omega$. This system is solved in a similar manner as our coupled system - that is, the first equation is advanced explicitly using Adams-Bashforth, and then information is transferred for use in the implicit solution by Newmark of the second equation. Numerical experiments of the coupled system (9) show a second-order temporal convergence as see in Fig. 3; see also [10].

Now that we have demonstrated that the simple contrived example for which we have an exact solution shows a second-order temporal convergence rate, we consider the FSI problem, with the proper transfer (projection) of data as will be explained in Section 5, and plot the difference in the plate's midpoint displacement (the midpoint displacement as computed by von-Kármán solver minus the one computed by $\mathscr{N} \varepsilon \kappa \mathscr{T}$ ar solver) for two different time steps: $\Delta t_{\mathrm{Nk}}=5 \times 10^{-5}$ and $\Delta t_{\mathrm{Nk}}=10^{-5}$ in Fig. 4 for 10 convective units. Recall that displacement is not transferred between the solvers, only velocity. Hence looking at the position of the wetted surface from the perspective of the fluid solver versus the perspective of the structural solver gives us some measure of the accuracy of the coupled system. Observe that the difference in the midpoint displacements is reduced by a factor of about 20 when the time difference is reduced by a factor of 5 , illustrating a second-order temporal convergence of the coupled system.

We can explain this behavior by examining the two time stepping schemes used for updating the displacement. Consider the following two equations:

$$
\begin{aligned}
& \frac{w_{\mathrm{F}}^{n+1}-w_{\mathrm{F}}^{n}}{\Delta t}=\frac{3}{2} \dot{w}_{\mathrm{F}}^{n}-\frac{1}{2} \dot{w}_{\mathrm{F}}^{n-1} \\
& \frac{w_{\mathrm{S}}^{n+1}-w_{\mathrm{S}}^{n}}{\Delta t}=\frac{1}{2}\left(\dot{w}_{\mathrm{S}}^{n+1}+\dot{w}_{\mathrm{S}}^{n}\right) .
\end{aligned}
$$

The first denotes second-order Adams-Bashforth as used by the fluid solver for updating the displacement from the fluid's perspective, and the second equation denotes a component of the Newmark scheme as used by the structural solver for updating the displacement from the structure's perspective. If we assume that the

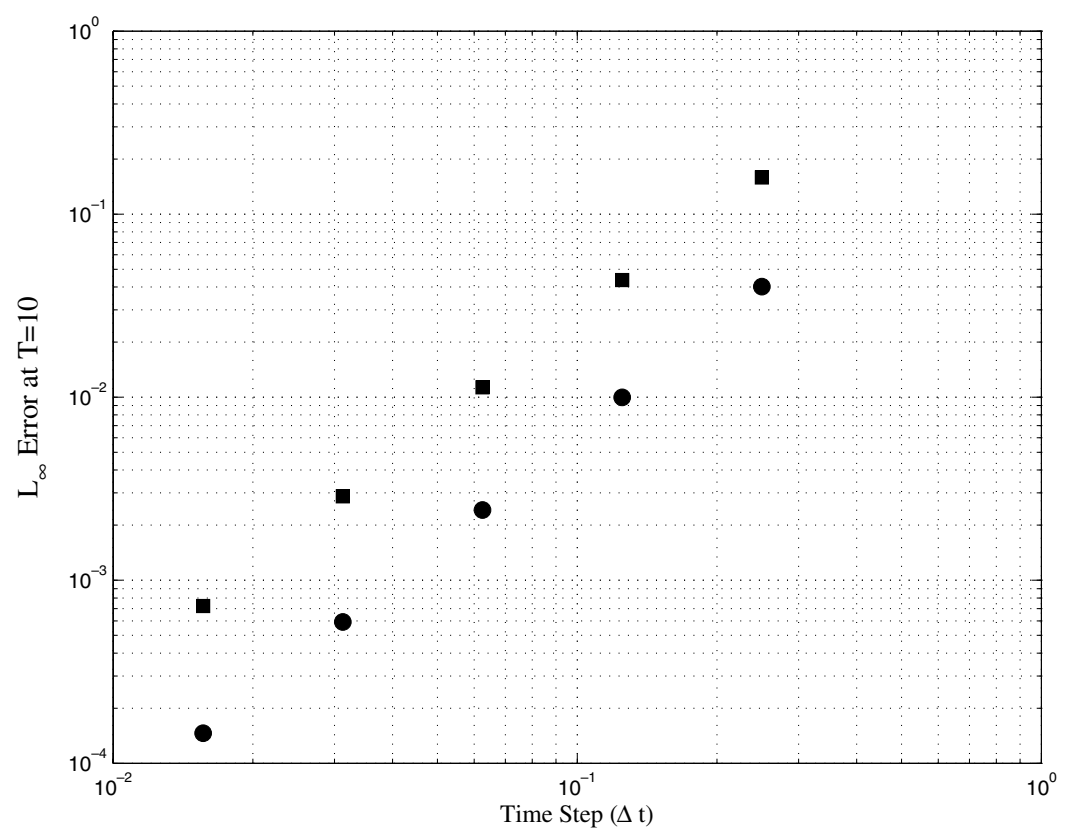

Fig. 3. The simple contrived linear system: $L_{\infty}$ error versus the $\Delta t$ for $\eta_{1}(t)$ (circles) and $\eta_{2}(t)$ (squares). The comparison is done at time $T=10$. 


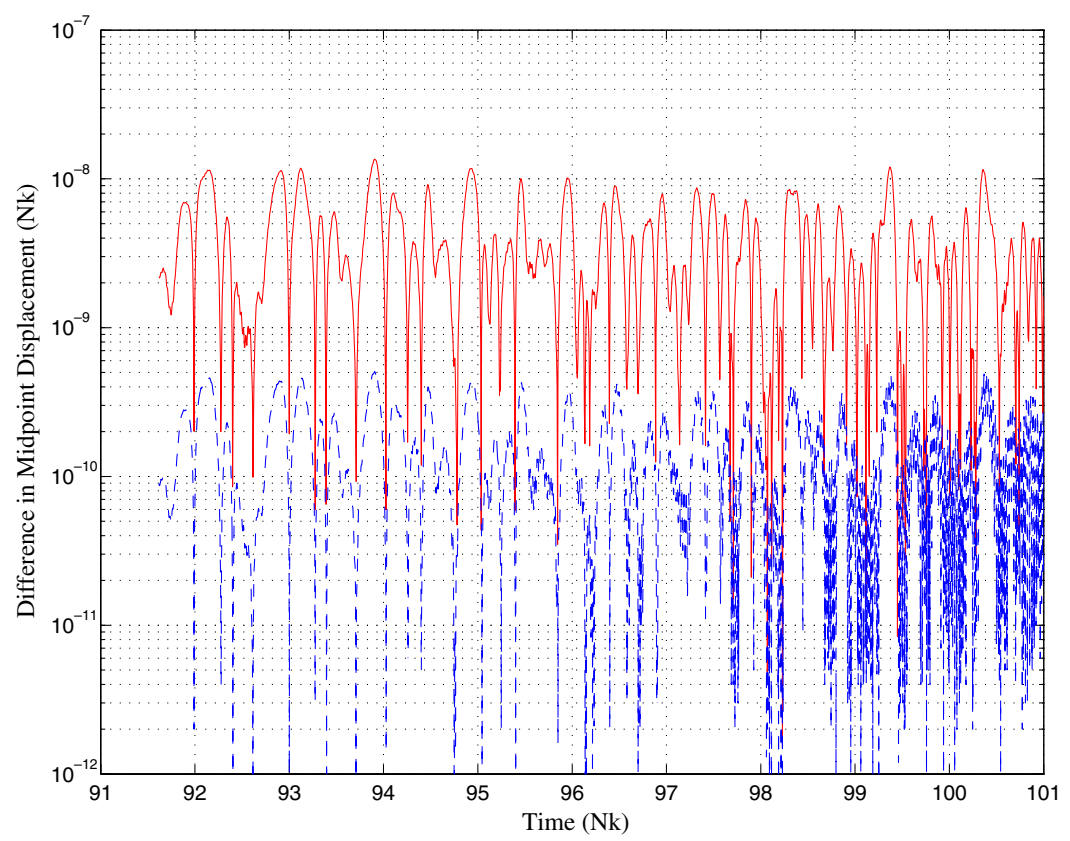

Fig. 4. Difference in the midpoint displacement as "seen" by von-Kármán and $\mathscr{N} \varepsilon \kappa \mathscr{T} \alpha r$ using conservative $L_{2}$ projections, and two different time steps: solid, $\Delta t_{\mathrm{Nk}}=5 \times 10^{-5}$; dashed, $\Delta t_{\mathrm{Nk}}=10^{-5}$.

interface velocity is identical on both the fluid and the structure, we can accomplish Taylor series on these two equations and compare terms to understand how the two displacements will differ with respect to time. After Taylor series, we see that the local difference between these two equations is of the form $C(\Delta t)^{3} \frac{\mathrm{d}^{3} w}{\mathrm{~d} t^{3}}(\xi)$ for a constant $C$ independent of the time step and some $\xi \in\left[t_{n}, t_{n+1}\right]$. Thus at any final time $T$ we would expect the difference between the displacement as seen by the fluid solver and as seen by the structural solver to be of order $\mathcal{O}\left(\Delta t^{2}\right)$. Note that this is the type of behavior we observe in Fig. 4.

\section{Spatial coupling strategy: how to transfer}

In addition to deciding what quantities to transfer and at what time instances to transfer, it is important to address the issue of how the transfer of quantities is accomplished. There has been a long-standing discussion of how to couple fluid and structural solvers when different meshes are used, as different meshing requirements exist for capturing the essential physics of the fluid and the structural systems (e.g. see $[11,22,34]$ ). In some cases, most of the instability in the coupled system can be attributed to how the interface variables were handled [35]. When high-order methods are employed, this "meshing" discrepancy has the possibility of being much larger, as different polynomial orders and inter-element continuity requirements may be used. Highorder methods used in this context force us to examine first-principles - in particular, the realization that differences in meshes as often discussed in the standard (low-order) finite element FSI literature is a special case of the more general problem of differences in the function spaces in which the solutions lie. The question of "how to transfer" generalizes to posing the following questions: (1) given knowledge of the function spaces used in the fluid and structural solvers, what projection operators should one employ for transferring data? and (2) what properties of the solution should one's projection operator preserve? For example, in [22], answers to these two questions are proposed for a class of scenarios involving the transfer of displacements and pressures. If one assumes a means of transferring displacements from the structure to the fluid and if one wants to conserve virtual work, then the algorithm in [22] dictates how pressure is to be transferred from the fluid to the structure. The approximation properties of the transfer of pressure are thus consequences of the first two choices: how one transferred displacements and the requirement of conservation of virtual work, and not of approximation requirements on pressure itself. The purpose of this section is to explore these issues 
in reference to our particular problem of interest, and to see what guiding principles can be deduced from the results of our case studies.

\subsection{Definitions of spaces used}

To set the stage for our case study results presented in the following section, it is necessary to understand the function spaces used in the fluid and structural solver and their relationship to each other. In Fig. 5 we present a diagram denoting the function spaces used by the two solvers and the required projection of the pressure and velocity between them.

First, let us comment upon the domains over which these function spaces act. In Section 2 we noted that, in general, the wetted surface from the fluid perspective and the structural perspective are different (i.e. $\Gamma_{\mathrm{FS}}^{\mathrm{F}}(t) \neq \Gamma_{\mathrm{FS}}^{\mathrm{S}}(t)$ for all time $\left.t>0\right)$. Thus the domain for $\mathscr{S}_{1}$ and $\mathscr{S}_{2}$ are different, a fact that must be taken into account when integrating functions from one space against another. In what follows, we will assume that the displacements are small, and thus we can define both the functions in $\mathscr{S}_{1}$ and $\mathscr{S}_{2}$ over the initial material configuration [0,2] $\times[0,2]$, which we will denote merely as $\Gamma_{\mathrm{FS}}$. If small displacements are not assumed, one must be careful to distinguish between functions in $\mathscr{S}_{1}$ over $\Gamma_{\mathrm{FS}}^{\mathrm{F}}$ and functions in $\mathscr{S}_{2}$ over $\Gamma_{\mathrm{FS}}^{\mathrm{S}}$. The integrals of quantities from the two distinct spaces can be accomplished using the common "pull-back" integration approach employed in non-linear solid mechanics [36]. We note that all integration accomplished in this paper was done numerically based upon common-refinement consistent integration [11]. In all computational experiments presented in this paper, $Q+1=9$ denoting that nine quadrature points/weights were used per direction per element in the common refinement. This was done as to guarantee exact (to machine precision) integration of the inner product of two polynomials of degree twelve.

The function space $\mathscr{S}_{1}$ denotes the space of all piecewise discontinuous polynomial functions based upon the mesh footprint of the fluid solver as shown in Fig. 6(left-bottom). For our particular case study, a $4 \times 4$ evenly-spaced patch of quadrilaterals was used, each quadrilateral supporting an $M$ th-order polynomial per direction. The pressure $p_{\mathrm{F}}(x, y)$ on the wetted surface as computed by the fluid solver is an element of $\mathscr{S}_{1}$, and can be written in terms of global basis functions $\phi(x, y): \Gamma_{\mathrm{FS}} \rightarrow \mathbb{R}, \mathscr{S}_{1}=\operatorname{span}\left\{\phi_{i}\right\}$ as follows:

$$
p_{\mathrm{F}}(x, y, z)=\sum_{i=0}^{M_{p}}\left(\hat{p}_{\mathrm{F}}\right)_{i} \phi_{i}(x, y)
$$

where $\left(M_{p}+1\right)$ denotes the total degrees of freedom used to express the pressure. In the simulations presented herein, pressure at the interface is calculated based upon the conservative variables used in the simulation and is projected back onto the same approximation space as the conservative variables.

The subspace of $\mathscr{S}_{1}$ in which the velocity of the interface resides is the set of all piecewise continuous $\left(C^{0}\right.$ continuous) polynomial functions based upon the mesh footprint. One can express the velocity of the interface $\dot{w}_{\mathrm{F}}(x, y)$ in terms of global basis functions $\psi_{i}(x, y): \Gamma_{\mathrm{FS}} \rightarrow \mathbb{R}$ as follows:

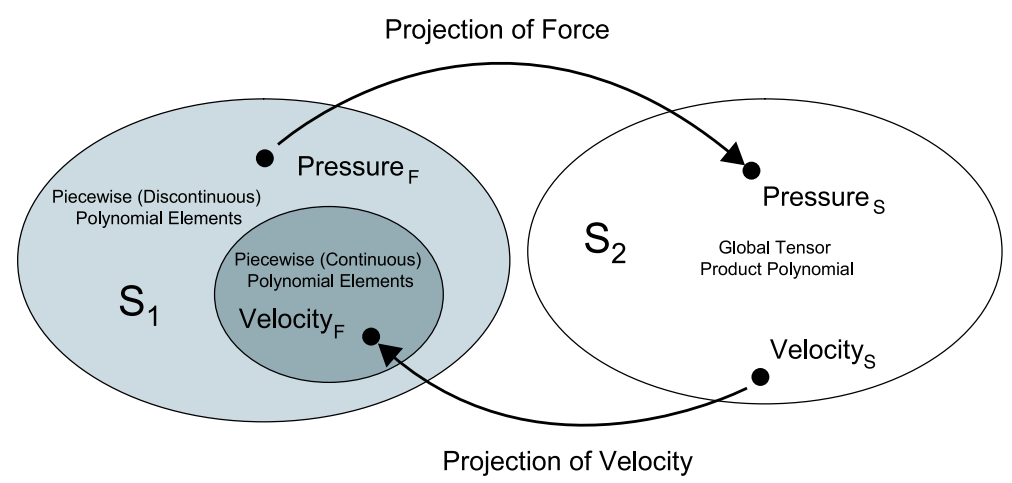

Fig. 5. Function spaces used by the two solvers under consideration and the required projection of the pressure and velocity between them. Explanation of the spaces is provided in the text. 

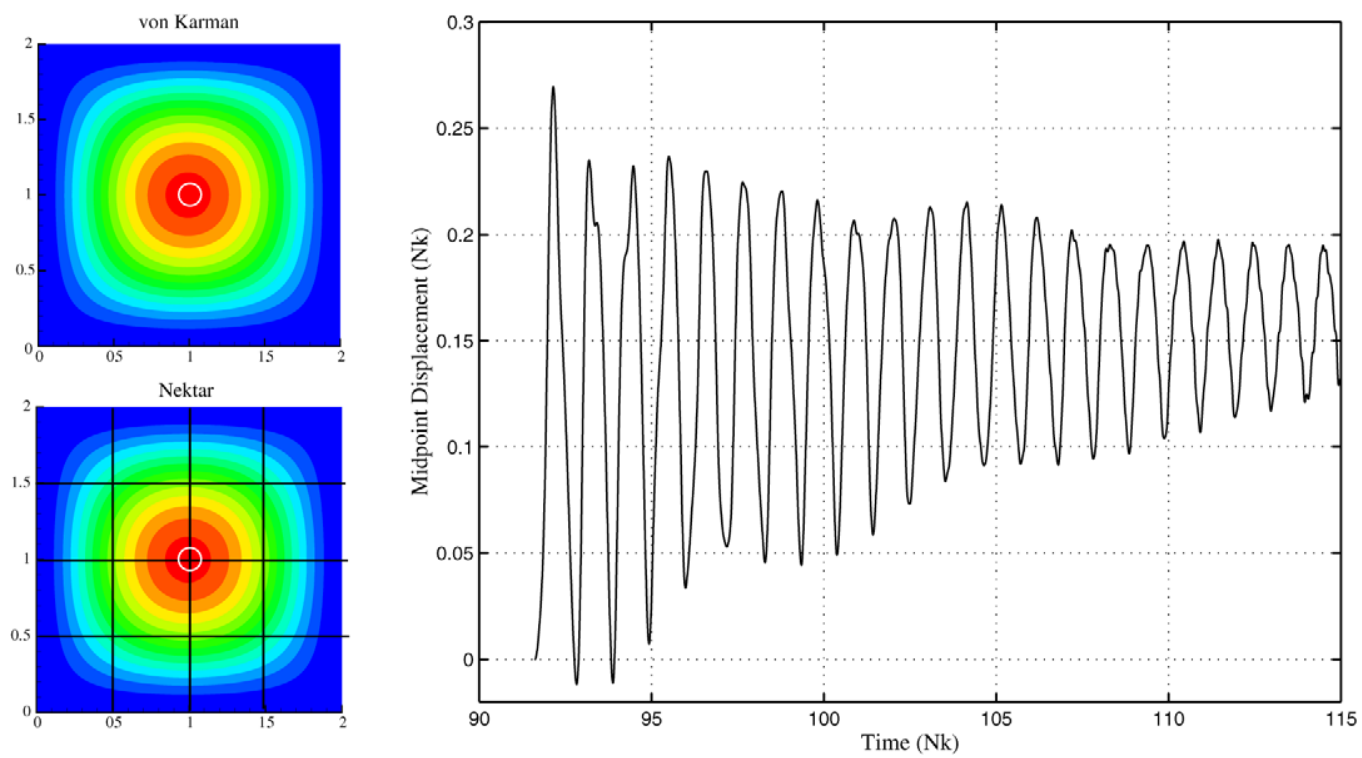

Fig. 6. The structural and fluid mesh footprints and deflection (left), and a typical plot showing the midpoint non-dimensionalized deflection as a function of time (right).

$$
\dot{w}_{\mathrm{F}}(x, y)=\sum_{i=0}^{M_{\mathrm{w}}}\left(\hat{\dot{w}}_{\mathrm{F}}\right)_{i} \psi_{i}(x, y)
$$

where $\left(M_{\mathrm{w}}+1\right)$ denotes the total degrees of freedom used to express the interface velocity, and where the basis functions $\operatorname{span}\left\{\psi_{i}\right\} \subseteq \operatorname{span}\left\{\phi_{i}\right\}$ have been chosen to automatically enforce $C^{0}$ continuity [9]. This space of functions is used to express both the wetted surface velocity and displacement from the fluid perspective. Note that this is not a problem with respect to the discontinuous Galkerin formulation due to the subspace relationship; it implies that the structural velocity inputted into the ALE formulation is already somewhat constrained with respect to the space of functions in which the fluid velocity resides.

The function space $\mathscr{S}_{2}$ denotes the space of all global tensor product polynomials constructed on the $[0,2] \times[0,2]$ square denoting the domain within the von-Kármán solver. As Chebyshev-collocation methods were used, this means that the pressure and velocity on the von-Kármán side can be expressed as follows:

$$
\begin{aligned}
& p_{\mathrm{S}}(x, y)=\sum_{i=0}^{N} \sum_{j=0}^{N}\left(\hat{p}_{\mathrm{S}}\right)_{i j} h_{i}(x) h_{j}(y) \\
& \dot{w}_{\mathrm{S}}(x, y)=\sum_{i=0}^{N} \sum_{j=0}^{N}\left(\hat{\dot{w}}_{\mathrm{S}}\right)_{i j} h_{i}(x) h_{j}(y)
\end{aligned}
$$

where $h_{i}(x)$ denotes the $N$ th-order Lagrange interpolating polynomial formed at the $(N+1)$ Chebyshev-Lobatto points $\left\{x_{j}\right\}$ with $h_{i}\left(x_{j}\right)=\delta_{i j}$. Hence, both the pressure and the velocity on the von-Kármán side are represented by global (over the wetted surface) $N$ th-order polynomial expansions (denoted at the tensor product polynomial space $\left.\mathscr{P}^{(N, N)}\right)$.

\subsection{Projection operators}

As denoted in Fig. 5, we must decide upon a projection operator to transfer pressure from the fluid to the structure $\left(p_{\mathrm{F}} \rightarrow p_{\mathrm{S}}\right)$ and velocity at the interface from the structure to the fluid $\left(\dot{w}_{\mathrm{F}} \rightarrow \dot{w}_{\mathrm{S}}\right)$. We consider three different projections for pressure (denoted PP-1 through PP-3) as follows: 


\subsubsection{PP-1: Discrete projection of pressure}

Procedure: For all quadrature points $\left(x_{i}, y_{i}\right) \in \Gamma_{\mathrm{FS}}, i=0, \ldots, N_{Q}$ in a consistent integration common refinement [11], sample the pressure using the native fluid discretization method to form the triplets $\left(x_{i}, y_{i},\left(p_{\mathrm{F}}\right)_{i}\right)$ where $\left(p_{\mathrm{F}}\right)_{i} \stackrel{\text { def }}{=} p_{\mathrm{F}}\left(x_{i}, y_{i}\right) \quad$ (notice that $\left(p_{\mathrm{F}}\right)_{i} \neq\left(\hat{p}_{\mathrm{F}}\right)_{i}$ in (12)). We define the vectors $\vec{p}_{\mathrm{F}} \stackrel{\text { def }}{=}\left(\left(p_{\mathrm{F}}\right)_{1},\left(p_{\mathrm{F}}\right)_{2}, \ldots,\left(p_{\mathrm{F}}\right)_{N_{Q}}\right)^{\mathrm{T}}$, and $\overrightarrow{\hat{p}}_{\mathrm{S}} \stackrel{\text { def }}{=}\left(\left(\hat{p}_{\mathrm{S}}\right)_{00}, \ldots,\left(\hat{p}_{\mathrm{S}}\right)_{(N+1),(N+1)}\right)^{\mathrm{T}}$. On the von-Kármán side, we may generate a linear system $\mathbf{A} \overrightarrow{\hat{p}}_{\mathrm{S}}=\overrightarrow{\vec{p}}_{\mathrm{F}}$ where the matrix $\mathbf{A}$ is a $N_{Q} \times(N+1)^{2}$ matrix defined by

$$
A_{i j}=h_{p}\left(x_{i}\right) h_{q}\left(y_{i}\right)
$$

where $i=0, \ldots, N_{Q}$ and $j=p+(N+1) q$, with $p, q=0, \ldots, N$. Note that the index $j$ is dependent on $p$ and $q$ - we could otherwise write the expression for the matrix as $A_{i, \tau(p, q)}$ where $\tau$ denotes an index map. In general, since $N_{Q} \gg(N+1)^{2}$, a rectangular system is formed. Multiplying both sides by the pseudo-inverse (i.e. $\left(\mathbf{A}^{\mathrm{T}} \mathbf{A}\right)^{-1} \mathbf{A}^{\mathrm{T}}$ ), we obtain the discrete least-squares projection of the pressure into the tensor product polynomial space used by the von-Kármán solver.

Comments: By sampling the pressure at a discrete number of points with no particular attention to the element boundaries within the fluid discretization (and the function space ramifications of those discontinuities in pressure), no particular properties (such as conservation) or features of the pressure are maintained. We are effectively treating the projection problem as a scattered data interpolation/projection problem. From the data fitting perspective, however, the pressure on the structure side "looks" very similar (in a collocation sense) to the pressure on the fluid side.

\subsubsection{PP-2: $L^{2}$ Projection of pressure}

Procedure: The goal is to find $p_{\mathrm{S}}(x, y) \in \mathscr{P}^{(N, N)}$ such that

$$
\int_{\Gamma_{\mathrm{FS}}} p_{\mathrm{S}}(x, y) h_{p}(x) h_{q}(y) \mathrm{d} A=\int_{\Gamma_{\mathrm{FS}}} p_{\mathrm{F}}(x, y) h_{p}(x) h_{q}(y) \mathrm{d} A
$$

for all $0 \leqslant p, q \leqslant N$. By substituting (14) for $p_{\mathrm{S}}$ and using our quadrature definition for integration we can generate a linear system $\mathbf{A} \overrightarrow{\hat{p}}_{\mathrm{S}}=\vec{b}$ where the $(N+1)^{2} \times(N+1)^{2}$ matrix and $(N+1)^{2} \times 1$ r.h.s vector are defined by:

$$
\begin{aligned}
A_{i j} & =\int_{\Gamma_{\mathrm{FS}}} h_{p}(x) h_{q}(y) h_{r}(x) h_{s}(y) \mathrm{d} A \\
b_{i} & =\int_{\Gamma_{\mathrm{FS}}} p_{\mathrm{F}}(x, y) h_{p}(x) h_{q}(y) \mathrm{d} A
\end{aligned}
$$

where $i=p+(N+1) q, j=r+(N+1) s, p, q, r, s=0, \ldots, N$. A is a symmetric positive definite matrix which can be inverted to solve for the coefficients $\left(\overrightarrow{\hat{p}}_{\mathrm{S}}\right)$. Again note the explicit expression of the index mapping in this expression.

Comments: The above formulation of the $L^{2}$ Galerkin projection is analogous to the consistent mass matrix formulation of projection (the approximation properties can be found in [9] and references therein). As such, it yields a function $p_{\mathrm{S}}(x, y)$ whose integral over the wetted surface is identical to the integral of $p_{\mathrm{F}}(x, y)$ over the wetted surface. Hence, this projection is total force preserving.

\subsubsection{PP-3: $L^{2}$ Constrained projection of pressure}

Procedure: The goal is to find $p_{\mathrm{S}}(x, y) \in \mathscr{P}^{(N C, N C)} \subseteq \mathscr{P}^{(N, N)}$ where $N C \leqslant N$, hence we are constraining the pressure to a subspace of the full $\mathscr{S}_{2}$. We accomplish the constrained $L^{2}$ projection as follows: Let

$$
p_{\mathrm{C}}(x, y)=\sum_{r=0}^{N C} \sum_{s=0}^{N C} \hat{p_{\mathrm{C} r s}} \tilde{h}_{r}(x) \tilde{h}_{s}(y)
$$

where $\tilde{h}_{r}(x)$ is a Lagrange interpolating polynomial based upon $N C+1$ Chebyshev-Gauss-Lobatto points and similarly for $\tilde{h}_{s}(y)$. We arrange $\hat{p_{\mathrm{C} r s}}$ into a vector of length $(N C+1) \times(N C+1)$ such that $\overrightarrow{\hat{p}_{\mathrm{C}}}=$ $\left(\hat{p_{\mathrm{C}} 00}, \ldots, \hat{p_{\mathrm{C} N C, N C}}\right)^{\mathrm{T}}$. We seek to find $p_{\mathrm{C}}(x, y)$ such that 


$$
\int_{\Gamma_{\mathrm{FS}}} p_{\mathrm{C}}(x, y)\left[\tilde{h}_{p}(x) \tilde{h}_{q}(y)\right] \mathrm{d} A=\int_{\Gamma_{\mathrm{FS}}} p_{\mathrm{F}}(x, y)\left[\tilde{h}_{p}(x) \tilde{h}_{q}(y)\right] \mathrm{d} A
$$

for all $0 \leqslant p, q \leqslant N C$. By substituting in (17) the expression for $p_{\mathrm{C}}(x, y)$ and using the quadrature definition for integration we can generate a linear system $\mathbf{A} \overrightarrow{\hat{p_{\mathrm{C}}}}=\vec{b}$ of the form:

$$
\begin{aligned}
A_{i j} & =\int_{\Gamma_{\mathrm{FS}}} \tilde{h}_{p}(x) \tilde{h}_{q}(y) \tilde{h}_{r}(x) \tilde{h}_{s}(y) \mathrm{d} A \\
b_{i} & =\int_{\Gamma_{\mathrm{FS}}} p_{\mathrm{F}}(x, y) \tilde{h}_{p}(x) \tilde{h}_{q}(y) \mathrm{d} A
\end{aligned}
$$

where $i=p+(N C+1) q, j=r+(N C+1) s, p, q, r, s=0, \ldots, N C$. The matrix $\mathbf{A}$ is a symmetric positive definite matrix which can be inverted to solve for the coefficients $\hat{p_{\mathrm{C} p q}}$. To find the coefficients of $p_{\mathrm{S}}(x, y)$ we now merely evaluate $p_{\mathrm{C}}(x, y)$ at the Chebyshev-Gauss-Lobatto points used to form the Lagrange interpolating functions of $p_{\mathrm{S}}$. Given the interpolating nature of $p_{\mathrm{S}}$ and the fact that $\mathscr{P}^{(N C, N C)} \subseteq \mathscr{P}^{(N, N)}$, we know that there is a unique set of coefficients $\hat{p}_{\mathrm{S}}$ such that $p_{\mathrm{vk}}(x, y)=p_{\mathrm{C}}(x, y)$ for all $(x, y) \in \Gamma_{\mathrm{FS}}$.

Comments: The constrained $L^{2}$ projection defined above has two important properties: (1) it is total force preserving and (2) it allows us to constrain the load exerted on the structure to a very small subspace of $\mathscr{S}_{2}$ without sacrificing conservation.

We also consider three different projections for velocity (denoted VP-1 through VP-3) as follows:

\subsubsection{VP-1: $H^{1 / 2}$ Projection of velocity}

Procedure: This projection procedure takes advantage of the hierarchical nature of the elemental basis used to construct the functions $\psi_{i}(x, y)$ as discussed in [9]. The basis functions $\psi_{i}(x, y)$ are generated by assembly from elemental basis functions $\psi_{j}^{e}(x, y)$ where $e$ denotes an element index and in which the assembly process enforces the $C^{0}$ continuity across interfaces. The elemental basis functions are hierarchical in that they are composed of vertex functions, edge functions and face functions (for the quadrilateral elements of interest to us for this work). An overview of the $H^{1 / 2}$ projection is as follows (for more details, consult [9, Chap. 2,4]): On an element $e$ within the footprint along the structure, set the vertex modes (which are interpolants of the solution) to the values of function being projected (in our case, of $\dot{w}_{\mathrm{S}}(x, y)$ ) at the nodes. After subtracting the vertex mode information, compute the edge modes of the solution by accomplishing the one-dimensional $L^{2}$ projection along each edge. After subtracting the edge mode information, compute the face modes of the solution by accomplishing an $L^{2}$ projection.

Comments: The advantage to this procedure is that it does not require the inversion of a "global" system formed by information over the entire wetted surface. Each element within the footprint of the mesh on the fluid side can be projected independent of its neighbor without sacrificing $C^{0}$ continuity. The disadvantage, however, is that the above method is not momentum preserving. There is no enforcement that the integral of $\dot{w}_{\mathrm{F}}$ over the interface constructed in this manner is equal to the integral of $\dot{w}_{\mathrm{S}}$.

\subsubsection{VP-2: $L^{2}$ Projection of velocity}

Procedure: The goal is to find $\dot{w}_{\mathrm{F}}(x, y) \in \operatorname{span}\left\{\psi_{j}\right\}$ such that

$$
\int_{\Gamma_{\mathrm{FS}}} \dot{w}_{\mathrm{F}}(x, y) \psi_{i}(x, y) \mathrm{d} A=\int_{\Gamma_{\mathrm{FS}}} \dot{w}_{\mathrm{S}}(x, y) \psi_{i}(x, y) \mathrm{d} A
$$

for all $0 \leqslant i \leqslant M_{\mathrm{w}}$. By substituting (15) for $\dot{w}_{\mathrm{F}}$ in (18), denoting the vector $\overrightarrow{\hat{\dot{w}}}_{\mathrm{F}}=\left(\left(\hat{\dot{\hat{w}}}_{\mathrm{F}}\right)_{0}, \ldots,\left(\hat{\dot{w}}_{\mathrm{F}}\right)_{M_{\mathrm{w}}}\right)^{\mathrm{T}}$, and using the quadrature definition for integration we can generate a linear system $\mathbf{A} \hat{\dot{\hat{w}}}_{\mathrm{F}}=\vec{b}$ of the form:

$$
\begin{aligned}
A_{i j} & =\int_{\Gamma_{\mathrm{FS}}} \psi_{i}(x, y) \psi_{j}(x, y) \mathrm{d} A \\
b_{i} & =\int_{\Gamma_{\mathrm{FS}}} \dot{w}_{\mathrm{S}}(x, y) \psi_{i}(x, y) \mathrm{d} A
\end{aligned}
$$

where $i, j=0, \ldots, M_{\mathrm{w}}$. The matrix $\mathbf{A}$ is a symmetric positive definite matrix which can be inverted to solve for the coefficients $\left(\hat{\dot{w}}_{\mathrm{F}}\right)_{j}$. 
Comments: Just as with the pressure, the above formulation of the $L^{2}$ projection is analogous to the consistent mass matrix formulation of projection (the approximation properties can be found in [9] and references therein). It provides a means of projecting the structural velocity into an appropriate function subspace (with respect to the fluid velocity function space) for use within the ALE formulation. As an $L^{2}$ projection, it yields a function $\dot{w}_{\mathrm{F}}(x, y)$ whose integral over the wetted surface is identical to the integral of $\dot{w}_{\mathrm{S}}(x, y)$ over the wetted surface. Hence, this projection is total momentum preserving.

\subsubsection{VP-3: $L^{2}$ Constrained projection of velocity}

Procedure: By constrained velocity projection, we mean that we first find the $L^{2}$ projection of the interface velocity $\dot{w}_{\mathrm{S}}$ to a subspace of $\mathscr{S}_{2}$ (i.e. to a tensor product polynomial over the entire wetted surface) and then $L^{2}$ project the intermediate representation to form $\dot{w}_{\mathrm{F}}$. The procedure is as follows: For $N C<N$ define

$$
\dot{w}_{\mathrm{C}}(x, y)=\sum_{r=0}^{N C} \sum_{s=0}^{N C}\left(\hat{\dot{w}}_{\mathrm{C}}\right)_{r s} \tilde{h}_{r}(x) \tilde{h}_{s}(y)
$$

where $\tilde{h}_{r}(x)$ is a Lagrange interpolating polynomial based upon $(N C+1)$ Chebyshev-Gauss-Lobatto points and similarly for $\tilde{h}_{s}(y)$. We arrange $\left(\hat{\dot{w}}_{\mathrm{C}}\right)_{r s}$ into a vector of length $(N C+1) \times(N C+1)$ such that $\overrightarrow{\hat{\dot{w}}}_{\mathrm{C}}=$ $\left(\left(\hat{\dot{w}}_{\mathrm{C}}\right)_{00}, \ldots,\left(\hat{\dot{w}}_{\mathrm{C}}\right)_{N C, N C}\right)^{\mathrm{T}}$. We now seek to find $\dot{w}_{\mathrm{C}}(x, y)$ such that

$$
\int_{\Gamma_{\mathrm{FS}}} \dot{w}_{\mathrm{C}}(x, y) \tilde{h}_{p}(x) \tilde{h}_{q}(y) \mathrm{d} A=\int_{\Gamma_{\mathrm{FS}}} \dot{w}_{\mathrm{S}}(x, y) \tilde{h}_{p}(x) \tilde{h}_{q}(y) \mathrm{d} A
$$

for all $0 \leqslant p, q \leqslant N C$. By substituting in (19) expression for $\dot{w}_{\mathrm{C}}(x, y)$ and using our quadrature definition for integration we can generate a linear system $\mathbf{A} \overrightarrow{\dot{w}}_{\mathrm{C}}=\vec{b}$ of the form:

$$
\begin{aligned}
& A_{i j}=\int_{\Gamma_{\mathrm{FS}}} \tilde{h}_{p}(x) \tilde{h}_{q}(y) \tilde{h}_{r}(x) \tilde{h}_{s}(y) \mathrm{d} A \\
& b_{i}=\int_{\Gamma_{\mathrm{FS}}} \dot{w}_{\mathrm{S}}(x, y) \tilde{h}_{p}(x) \tilde{h}_{q}(y) \mathrm{d} A
\end{aligned}
$$

where $i=p+(N C+1) q, j=r+(N+1) s, p, q, r, s=0, \ldots, N C$. The matrix A is a symmetric positive definite matrix which can be inverted to solve for the coefficients $\left(\hat{\dot{w}}_{\mathrm{C}}\right)_{p q}$. The function $\dot{w}_{\mathrm{C}}(x, y) \in \mathscr{P}^{(N C, N C)} \subseteq \mathscr{S}_{2}$ represents the $L^{2}$ projection of the interface velocity before transferring it to the fluid. To find the function $\dot{w}_{\mathrm{F}}(x, y)$, the $L^{2}$ projection method (VP-2) defined above is used with the replacement of $\dot{w}_{\mathrm{C}}(x, y)$ for $\dot{w}_{\mathrm{S}}$.

Comments: The constrained $L^{2}$ projection defined above essentially acts as a filter of the velocity before transferring it to the fluid discretization. By projecting down to a subspace of $\mathscr{S}_{2}$ we reduce the oscillatory nature of the solution. Note, however, that since $L^{2}$ projections are used, the momentum is conserved. That is, although the velocity is "filtered", it is still true that the integral of $\dot{w}_{\mathrm{F}}(x, y)$ wetted surface is identical to the integral of $\dot{w}_{\mathrm{S}}(x, y)$ over the wetted surface.

\subsection{Conservation}

Following [15], let us define metrics for evaluating our temporal and spatial coupling algorithms. Given the definitions for pressure and velocity above, we can define the following desired conserved quantities on the interface:

$$
\begin{aligned}
& \text { Conservation of total force : } \int_{\Gamma_{\mathrm{FS}}} p_{\mathrm{F}} \mathrm{d} A=\int_{\Gamma_{\mathrm{FS}}} p_{\mathrm{S}} \mathrm{d} A \quad \forall t_{n} \\
& \text { Conservation of momentum : } \int_{\Gamma_{\mathrm{FS}}} \dot{w}_{\mathrm{F}} \mathrm{d} A=\int_{\Gamma_{\mathrm{FS}}} \dot{w}_{\mathrm{S}} \mathrm{d} A \quad \forall t_{n} \\
& \text { Conservation of energy of ext. forces : } \int_{\Gamma_{\mathrm{FS}}} p_{\mathrm{F}} w_{\mathrm{F}} \mathrm{d} A=\int_{\Gamma_{\mathrm{FS}}} p_{\mathrm{S}} w_{\mathrm{S}} \mathrm{d} A \quad \forall t_{n}
\end{aligned}
$$

The first equation represents the conservation of the total force due to pressure forces from the fluid; it guarantees that the force supplied by the fluid is (in an integral sense) the external loading experienced by the struc- 
ture. The second equation represents the conservation of interface momentum under the assumption of constant density; it guarantees that the momentum at the interface as computed by the structure is properly experienced (in the integral sense) by the fluid. The third equation represents the conservation of the energy due to the external forces, also viewed as the work at the interface; it guarantees that work expended by the structure is transferred to the fluid and vise versa.

The choice of projection mechanisms directly impact which of these quantities are conserved. For instance, as previously noted, the algorithm in [22] conserves virtual work (i.e. energy due to external forces) in space [15] at the sacrifice of conserving total momentum and total force. Since we pass velocities instead of displacements and hence cannot directly follow the guidance of [22], we have chosen to attempt to conserve total force and total momentum. As a consequence of our decision, we will not conserve virtual work (in space) when we transfer quantities. We thus define the difference in the energy of external forces over the wetted surface as [15]:

$$
\Delta_{\mathrm{wet}} E^{(\mathrm{ex})}(t) \stackrel{\text { def }}{=} \int_{\Gamma_{\mathrm{FS}}} p_{\mathrm{S}} w_{\mathrm{S}} \mathrm{d} A-\int_{\Gamma_{\mathrm{FS}}} p_{\mathrm{F}} w_{\mathrm{F}} \mathrm{d} A,
$$

a quantity which we will measure as part of our simulation.

\section{Numerical Examples}

Herein we present numerical results obtained by the different projection algorithms described in the previous section, conserving different quantities (e.g. total force, momentum). The notion of "blow up" due to an improper transfer of data between the two codes is also addressed, shown not to be directly associated with conservation of energy, but because of aliasing errors induced in the fluid because of oscillations in velocities passed to the fluid code. This phenomenon occurs because of projecting a discontinuous pressure from the flow to a high order polynomial space representing the von-Kármán plate model, which in turn yields an oscillatory velocity field passed back to the fluid thus causing aliasing modes to be excited and hence "blow up" of the fluid solver.

\subsection{Parameters used in case study}

For the computational experiments, we consider a 3-D viscous compressible flow over a flexible plate embedded in a wall as shown in Fig. 1(left). The computational domain is 26 units in the streamwise direction, 22 units in the crossflow direction and 2 units in the wall normal direction. For the inflow conditions, a laminar boundary layer profile corresponding to Blasius flow one unit downstream is used [37]. Periodic conditions are used on the sides of the domain, and freestream conditions are enforced on the top of the domain. Wall boundary conditions satisfied no-slip and no penetration. A Reynolds number of $R e_{\infty}=10,000$ with respect to half the plate length and a Mach number of $M a_{\infty}=0.2$ were used.

For the structure, a square plate of dimensions $[0,2] \times[0,2]$, having a thickness of $h=0.1$, and a structural damping coefficients of $c=1.0$ and Poisson ratio of $v=0.3$ were used. The boundary conditions for the plate are clamped (zero displacement and zero slope for all time). Unless otherwise stated, a mass ratio of $M R=100$ and rigidity ratio of $R R=10,000$ were used in all simulations.

The fluid domain $\Omega_{\mathrm{F}}(t)$ is discretized with 720 hexahedral elements having a distribution as indicated in Fig. 1. For most numerical experiments, third-order polynomials per direction were used in the fluid solver; however, experiments up to seventh-order polynomials in the fluid were accomplished to verify the consistency of the results presented.

The structural domain was represented by a $[0,2] \times[0,2]$ plate. The tensor product of Lagrange polynomials on the Chebyshev points were used as the basis for all quantities within the von-Kármán solver. Most numerical experiments were accomplished with the tensor product of twelfth-order polynomials (i.e. 13 points per direction), however experiments were done with different orders to verify the consistency of the results.

The wetted surface from the structural solver consisted of a single quadrilateral domain as seen in Fig. 6(left-top). From the perspective of the fluid solver, the interface consisted of a mesh footprint containing 16 evenly-spaced quadrilateral elements as shown in Fig. 6(left-bottom). In both figures, we present a contour plot of the representative deflection of the interface from the initial (flat plate) configuration. In Fig. 6(right) 
we plot the midpoint deflection (deflection at the white circles in Fig. 6(left top and bottom)) as a function of $t_{\mathrm{F}}$. Plots of this form will be used throughout the next two sections. For all plots, we have used the conversion factors presented in Table 3 to present all quantities in $\mathscr{N} \varepsilon \kappa \mathscr{T} \alpha r$ (i.e. fluid discretization) non-dimensionalization units (denoted by $F$ on the plots).

Notice that once the non-dimensionalized time-step $\Delta t$ is chosen in the fluid solver, the time-step in the solid solver is uniquely determined so the "physical" instance at which information is passed between solvers is identical (see Appendix C).

We initiate the fluid solver to obtain a steady state flow, keeping the plate at its position (as a rigid solid) up to the state of about $t_{\mathrm{F}}=90 \mathscr{N} \varepsilon \kappa \mathscr{T} \alpha r$ time units, at which time we "free" the plate to represent actual elastic response. Thus all results are shown starting at $t_{\mathrm{F}}=90 \mathscr{N} \varepsilon \kappa \mathscr{T} \alpha r$ time units.

\subsection{Projection methods for stability: case study 1}

In the following case study, third-order polynomials were used within the fluid discretization, twelfth-order Chebyshev polynomials were used within the structural discretization, and a time step of $\Delta t_{\mathrm{F}}=5 \times 10^{-5}$ was used unless we specifically state otherwise.

The collection of cases we investigated in this subsection is provided herein (with a summary of the results provided in Table 1):

Case 1. A: Non-conservative in force and velocity: We started our investigation by using discrete projection for pressure (PP-1) and the $H^{1 / 2}$ projection for velocity (VP-1). Within approximately 25 units the solution blows up.

Case 1.B: Conservative in force but non-conservative in velocity: Under the assumption that conservation of forces was probably the problem in Case 1.A, we switched to using an $L^{2}$ projection for pressure (PP-2) and the $H^{1 / 2}$ projection for velocity (VP-1). Within approximately 10 units the solution blows up. For demonstration purposes we present in Fig. 7 the difference in force and momentum over the wetted surface for this case (showing a conservation of these quantities), and in Fig. 8 the midpoint displacement and difference in external forces energy over the wetted surface. At a time instance shortly before the solution blows up, we extract the pressure field and the structural velocity field as seen by the structure (shown in the Fig. 7 inset plots, top and bottom, respectively). Note that although the total force is conserved due to the use of the PP-2 conservative projection, this does not enforce that the pressure projection is good from an approximation theory perspective. Also note that the idea of blow-up appears to be related to the spatial variations in pressure and velocity (a point we will explore further).

Case 1.C: Non-conservative in force but conservative in velocity: Given that conservation of forces seemed insufficient, we examined the other permutation - namely, we used the discrete projection for pressure (PP-1) with the conservative $L^{2}$ projection for velocity (VP-2). Within approximately 12 units the solution blows up.

Case 1.D-Case 1.F: Conservative in force and in velocity: Under the assumption that conserving total force without conserving total momentum (or vice versa) was insufficient, we switched to using an $L^{2}$ projection for pressure (PP-2) and an $L^{2}$ projection for velocity (VP-2) - denoted by case Case 1.D. Within approx-

Table 1

Summary of case study 1: projections and results

\begin{tabular}{lllll}
\hline Case & Pressure projection & Velocity projection & Result & Comment \\
\hline 1.A & PP-1 & VP-1 & Unstable & Blowup after $\approx 25$ units \\
1. B & PP-2 & VP-1 & Unstable & Blowup after $\approx 10$ units \\
1. C & PP-1 & VP-2 & Unstable & Blowup after $\approx 12$ units \\
$1 . D$ & PP-2 & VP-2 & Unstable & Blowup after $\approx 12$ units \\
$1 . \mathrm{E}$ & PP-2 & VP-2 & Unstable & Blowup after $\approx 10$ units \\
$1 . F$ & PP-2 & VP-2 & Unstable & Blowup after $\approx 10$ units \\
$1 . \mathrm{G}$ & PP-1 & VP-3 & Stable & Stable for over 50 units \\
$1 . \mathrm{H}$ & PP-2 & VP-3 & Stable & Stable for over 50 units \\
\hline
\end{tabular}



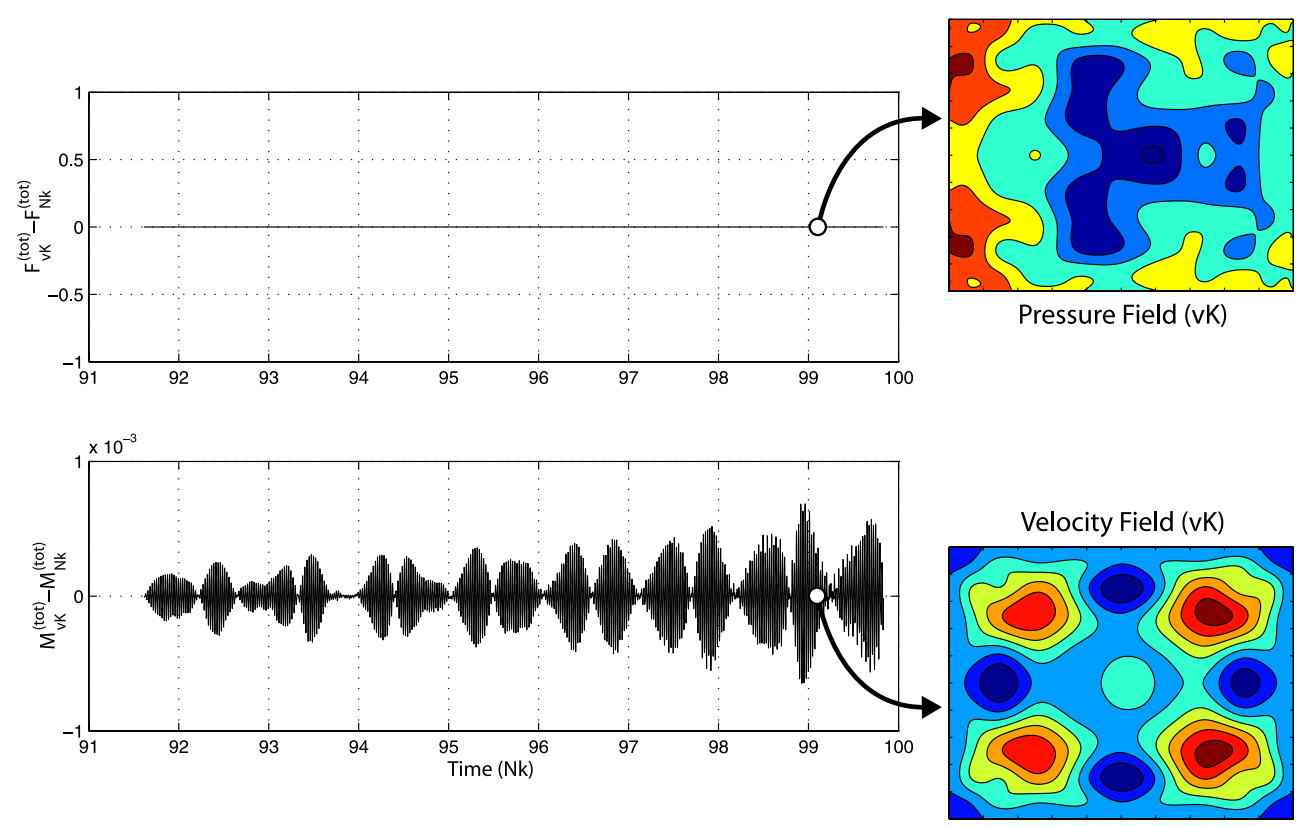

Fig. 7. Case 1.B: Difference in force (top) and difference in momentum over the wetted surface. $L^{2}$ projection of pressure (PP-2) and $H^{1 / 2}$ projection of velocity (VP-1). The pressure field and velocity field at a time instant prior to blow-up are shown as inset plots.
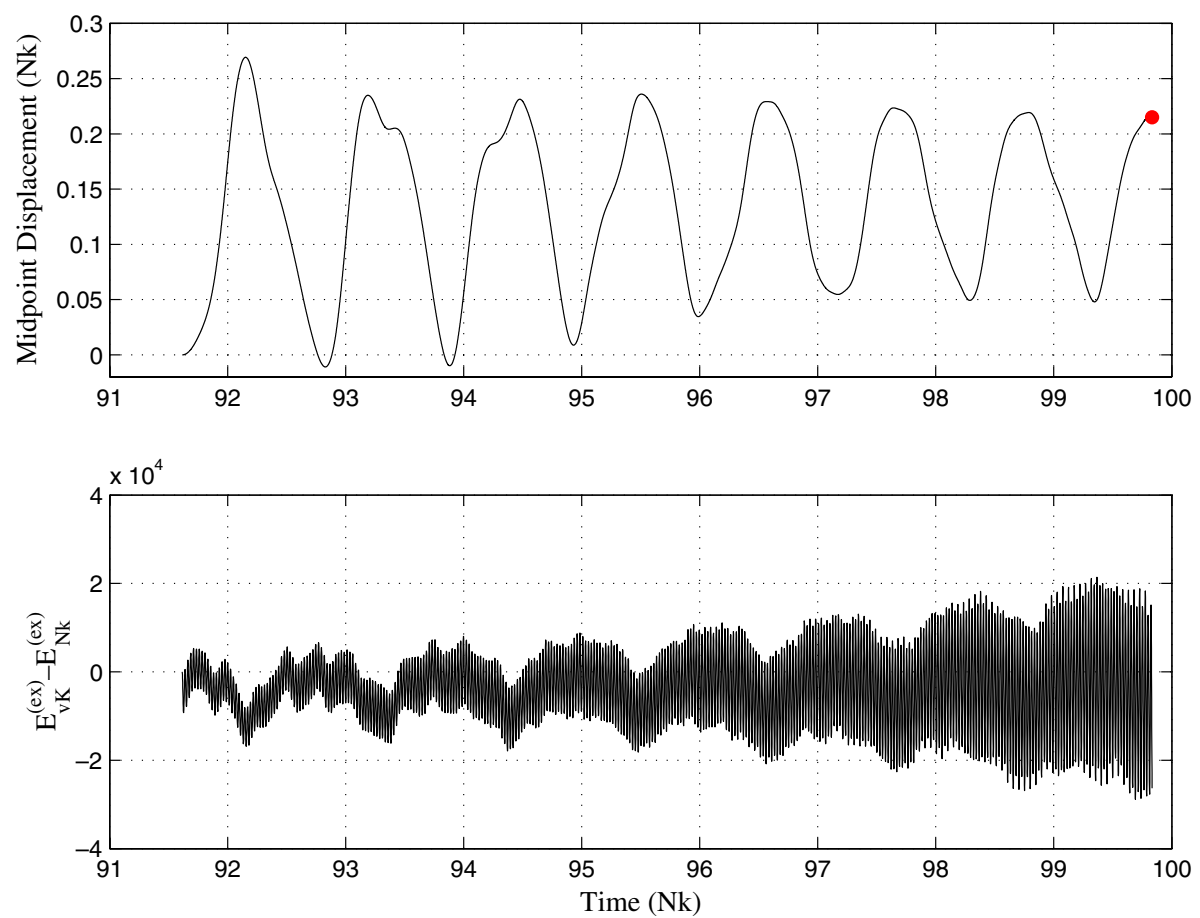

Fig. 8. Case 1.B: The midpoint displacement (top) and difference in external forces energy over the wetted surface $\Delta_{\text {wet }} E^{\text {ex }}$ (bottom). The circle in the top graph represents the "blowup" of the results. PP-2/VP-1 projections.

imately 12 units the solution blows up. Somewhat discouraged by the fact that our conservative projections did not solve the instability problem, we tried lowering the time step to $1.0 \times 10^{-5}$ to see if the solution would become stable (Case 1.E). Within approximately 10 units the solution blows up. To eliminate the 
possibility of the problem being under-resolution in the fluid, we tried increasing the polynomial order of the fluid discretization to fifth-order and maintained the small time step of $1.0 \times 10^{-5}$ (Case 1.F). Within approximately 10 units the solution blows up.

Case 1.G-Case 1.H: Conservative and non-conservative in force and constrained-conservative in velocity: Having examined plots of the velocity of the interface at various time instances, we noticed that the interface velocity field quickly appeared highly undulating. We hence postulated that not only conservation of forces and momentum were necessary but that also some filtering of the velocity was also necessary. We switched to using an $L^{2}$ projection for pressure (PP-2) and a constrained $L^{2}$ projection for velocity (VP-3) in which we projected down to third-order tensor products within $\mathscr{S}_{2}$ before passing information to the fluid solver (Case 1.G).

With this modification, the solution remained stable for long time. In Fig. 9 we present the midpoint displacement (top) and the difference in external force energy over the wetted surface (bottom). To understand whether conservation or constraining the velocity was more important, we also ran a simulation using the discrete projection for pressure (PP-1) and the constrained $L^{2}$ projection for velocity (VP-3) (Case 1.H). It also was stable for long time. The midpoint displacement was nearly identical to that of Case 1.G; however, the difference in external forces energy over the wetted surface $\left(\Delta_{\text {wet }} E^{\mathrm{ex}}\right)$ was three orders of magnitude larger $\left(\mathcal{O}\left(10^{-4}\right)\right.$ for Case $1 . \mathrm{H}$ versus $\mathcal{O}\left(10^{-7}\right)$ for Case $\left.1 . \mathrm{G}\right)$.

\subsection{Projection methods for both stability and accuracy: case study 2}

Although a stable solution was obtained, it was somewhat disconcerting that "filtering" (constrained projections) were needed. This led us to the natural question of what ramification on the midpoint displacement did the constrained projection of the velocity have. In Fig. 10 we compare the Case 1.D ("unfiltered" but unstable) with Case 1.G (stable but "filtered").
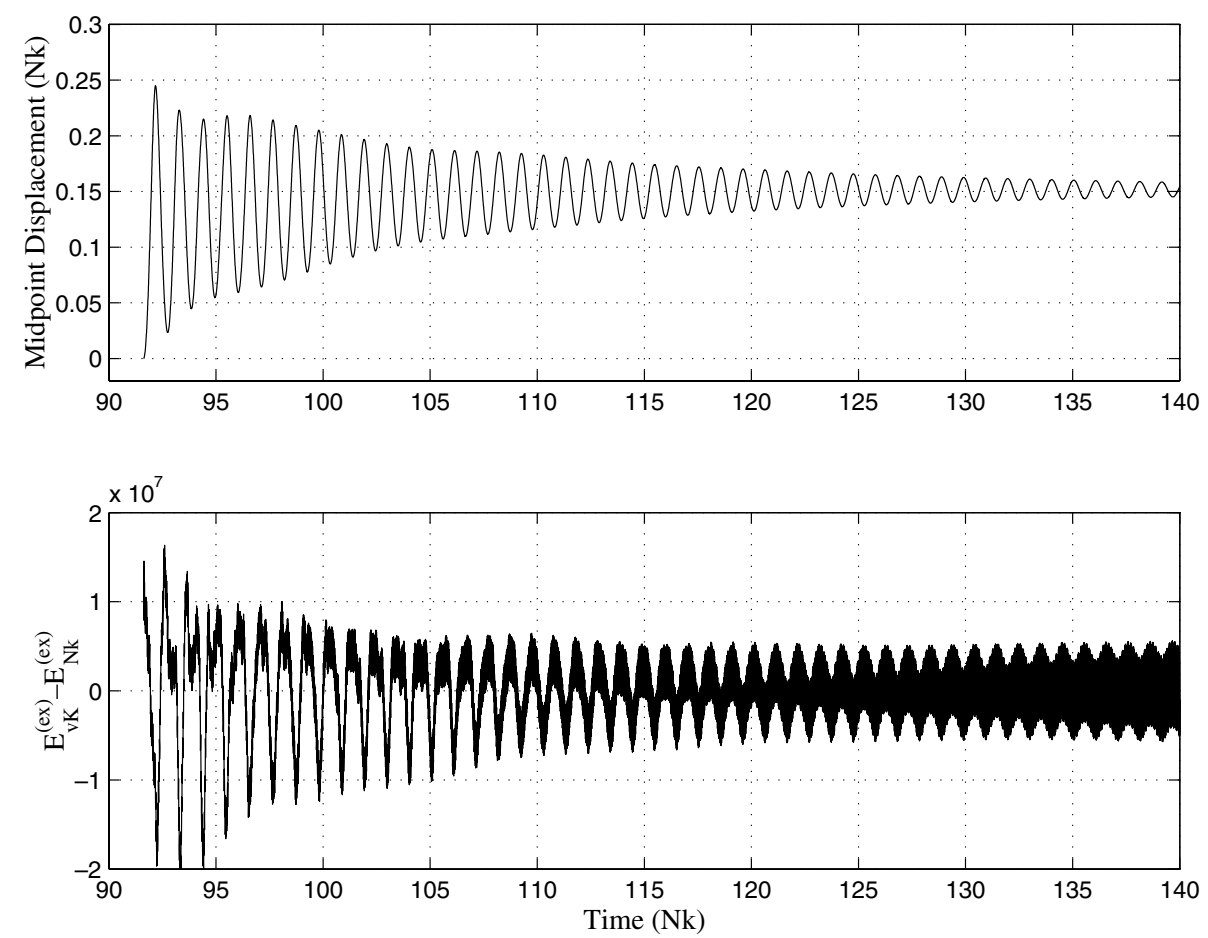

Fig. 9. Case 1.F: PP-2/VP-3 projections ( $L^{2}$ projection of pressure and constrained - projected to 2nd-order on the von-Kármán side - $L^{2}$ projection of velocity). The midpoint displacement (top) and difference in external forces energy over the wetted surface $\Delta_{\text {wet }} E^{\text {ex }}$ (bottom). 


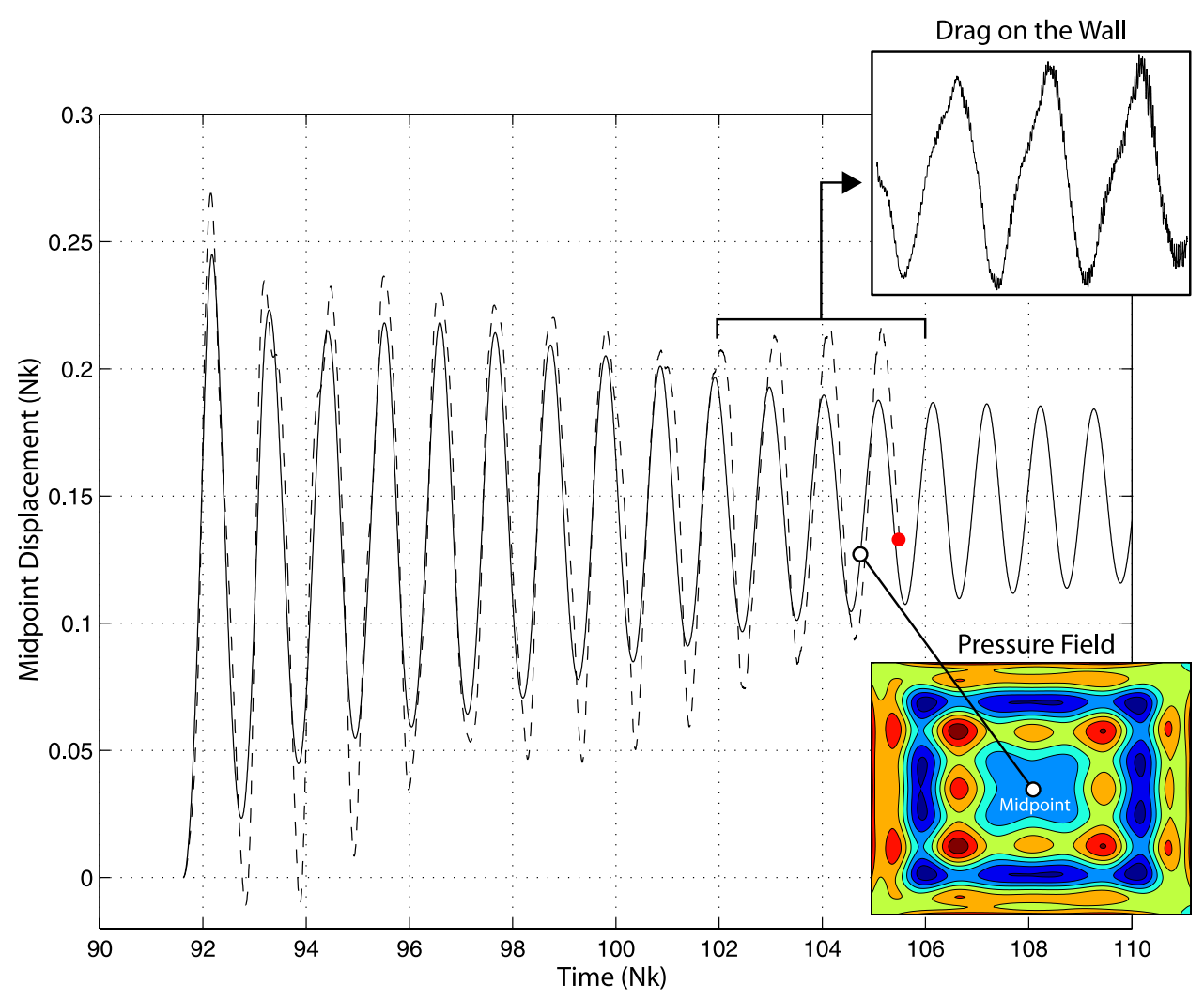

Fig. 10. The midpoint displacement for a stable but "filtered" coupling compared to an unstable but "unfiltered" coupling. Solid line - Case 1-G: Stable but "filtered" (PP-2/VP-3); Dash line - Case 1-D: "Unfiltered" but unstable (PP-2/VP-2). The circle denotes the place of blowup for Case 1-D. We provide inset plots of the drag on the wall and the pressure field at a particular time instance just prior to blow-up.

Observe in Fig. 10 that a discrepancy exists between the midpoint displacements of the "filtered" and "unfiltered" solutions. Although stability was achieved, variation in the midpoint displacement forced us to reexamine our assumption of filtering the interface velocity.

Remark 5. In Fig. 10 we provide a inset plot of the drag on the wall - a integrated quantity of the forces on the wall. This demonstration gives further hint that the instability might not be due to an energy imbalance on the interface but rather due to the projection properties of pressure and/or velocity.

We next examined the pressure we were inputting into the structural solver, as this solver inputs pressure and outputs structural velocity. When doing so, an interesting phenomenon is noticed: in Fig. 11 we present on the top row a collection of snapshots of the projected pressure as seen by the von-Kármán solver at various time instances. Note the rather non-smooth nature of the pressure. In the lower row, we show a constrained pressure projection (PP-3) in which we constrain the pressure to be quadratic over the surface of the plate. The latter contains far less wiggles.

From the above results we concluded that the projection of the discontinuous pressure (discontinuous at element interfaces due to the use of the DG formulation) onto the continuous (global) tensor project representation used by the fluid introduced oscillations (Gibbs phenomenon). Hence, the system was driven unstable. Although we were respecting the conservation of total force and total momentum (which many claim is desirable and even necessary), we were not respecting the inherent properties of the function spaces. This problem of subspace mismatch is accentuated due to the non-monotonicity preserving nature of high-order methods, as conservative $L^{2}$ projections can introduce wiggles when a fundamental assumption concerning the relationship of the spaces is violated (for instance, attempting to represent a discontinuous function with a polynomial function). 


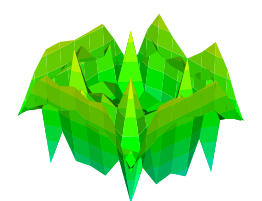

$\mathrm{t}_{\mathrm{Nk}}=102.415$

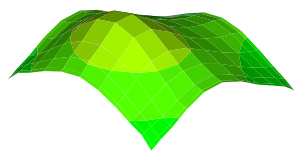

$\mathrm{t}_{\mathrm{Nk}}=102.415$

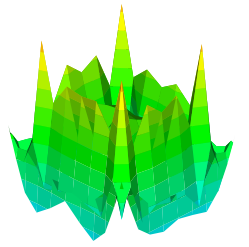

$\mathrm{t}_{\mathrm{Nk}}=103.465$

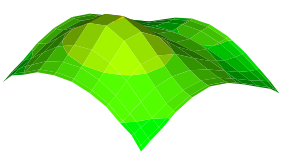

$\mathrm{t}_{\mathrm{Nk}}=103.465$

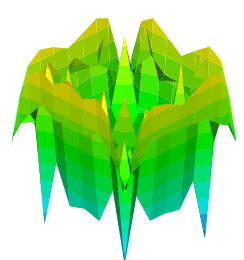

$\mathrm{t}_{\mathrm{Nk}}=104.465$

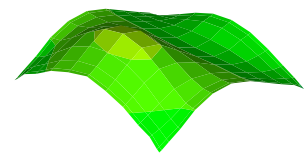

$\mathrm{t}_{\mathrm{Nk}}=104.465$
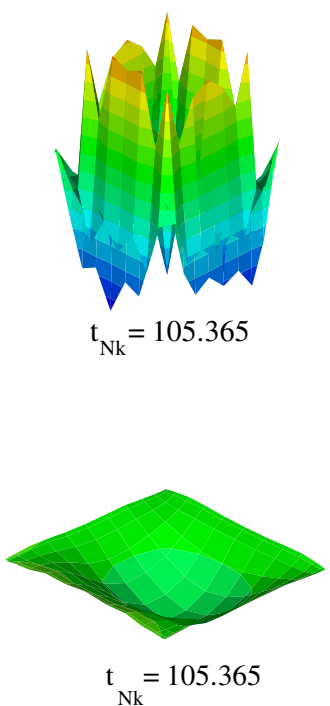

Fig. 11. Snapshots of the projected pressure distribution "as seen" by the von-Kármán solver (i.e. $p_{\mathrm{S}}(x, y)$ ) as a function of $t_{\mathrm{F}}$. Top snapshots represent use of the $L^{2}$ projection (PP-2); Bottom snapshots represent the constrained $L^{2}$ projection (PP-3).

Remark 6. The above conclusion is very similar to what is seen in mortar element methods [38-40]. It is insufficient to merely define "conservative" projections, but one must understand the relationships between the function spaces in order to determine what other properties to mandate. In fact, one could argue that compliance with global conservation properties is a secondary consideration - the primary constraint on projections should be maintaining the trace-space relations.

Remark 7. The problem experienced above is not fundamentally due to our use of the discontinuous Galerkin method. The same type of behavior may be observed when two different $C^{0}$ high-order finite element meshes are interfaced. If the $C^{0}$ interface on one mesh lies within the interior of a high-order element on the other mesh, Gibbs phenomena will occur unless special care is taken to limit oscillations. Low-order finite element methods do not experience this problem due to the monotonic nature of the (linear) basis functions. Hence, it is the classic trade-off of accuracy (i.e. ability to represent the solution) versus monotonicity. To demonstrate this, consider the example given in Fig. 12. On the left, we show the projection of a snapshot (in one dimension) of the discontinuous pressure field on to a $C^{0}$ high-order finite element basis. Note, as we would expect from approximation theory [41], oscillations develop in the region of the discontinuity. On the right we show what happens if we project information from one $C^{0}$ high-order finite element mesh on to another $C^{0}$ high-
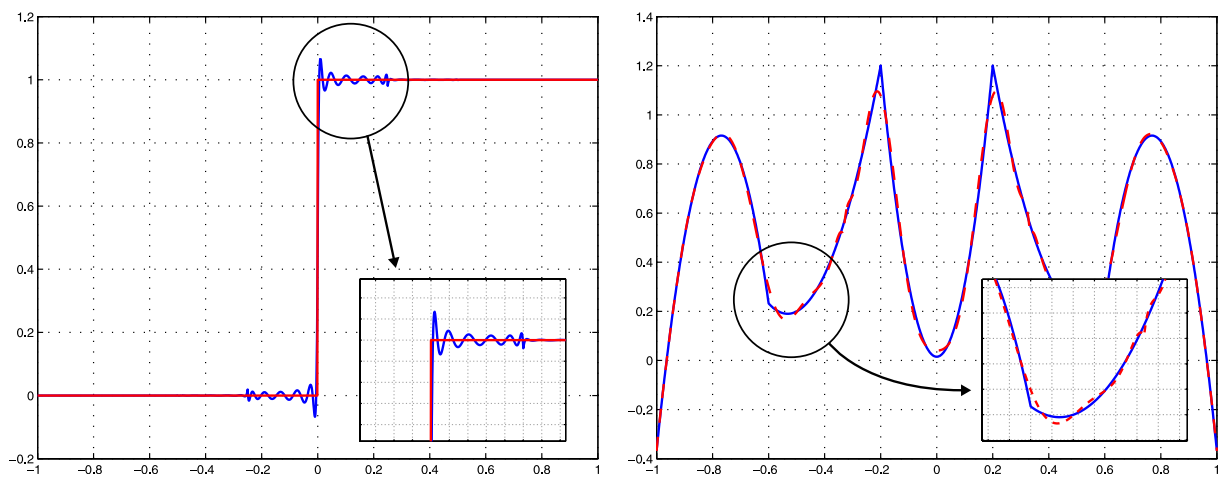

Fig. 12. On the left plot we show the result of accomplishing an $L_{2}$ projection of the Heavi-side function on to a high-order $C^{0}$ finite element mesh; on right we show the result of projecting a function from one high-order $C^{0}$ finite element mesh to another high-order $C^{0}$ finite element mesh in which the element interfaces are not aligned. 
order finite element mesh. We have specifically placed the element interfaces of the second mesh in the middle of the first mesh. Note that the projection (shown as the dashed red line) is not monotonic - it oscillates around the places where the element breaks occur. Although the projection is conservative in the global sense, it introduces spatial variations which can cause problems.

Three new test cases (labeled Case 2.A-2.C) were performed to verify our statements above. A summary of the results is provided in Table 2. For Case 2.A-2.C, a constrained-conservative projection in force (PP-3) and conservative projection in velocity (VP-2) were used; the fluid discretization was maintained at third-order polynomials, the structural discretization was maintained at twelfth-order polynomials, and the time step was taken as $\Delta t_{\mathrm{F}}=5.0 \times 10^{-5}$. In Case 2.A, we projected the force to a time-dependent constant function. In Case 2.B, we projected the force to a time-dependent quadratic function; a representative plot of midpoint displacement and difference in external energy is presented in Fig. 13. In both cases, the solution remained stable for long time. To further verify our results, as Case 2.C, we increased the polynomial order of our fluid discretization to fifth-order, decreased the time step to $\Delta t_{\mathrm{F}}=1.0 \times 10^{-5}$, and projected the force to a timedependent quadratic function; the solution remained stable for long time.

In Fig. 14 we revisit the midpoint displacement comparison accomplish previously in Fig. 10. Recall that although a stable solution (dashed) was obtained when we first projected the velocity from 12th-order to

Table 2

Summary of case study 2 : projections and results.

\begin{tabular}{lllll}
\hline Case & Pressure projection & Velocity projection & Result & Comment \\
\hline 2.A & PP-3 (time dependent constant) & VP-2 & Stable & Stable for over 50 units \\
2.B & PP-3 (time dependent quadratic function) & VP-2 & Stable & Stable for over 50 units \\
2.C & PP-3 (time dependent quadratic function) & VP-2 & Stable & Stable for over 50 units \\
\hline
\end{tabular}

${ }^{\text {a }}$ Fifth-order spatial discretization of $\mathscr{N} \varepsilon \kappa \mathscr{T} \alpha r$ with $\Delta t_{\mathrm{F}}=1.0 \times 10^{-5}$.
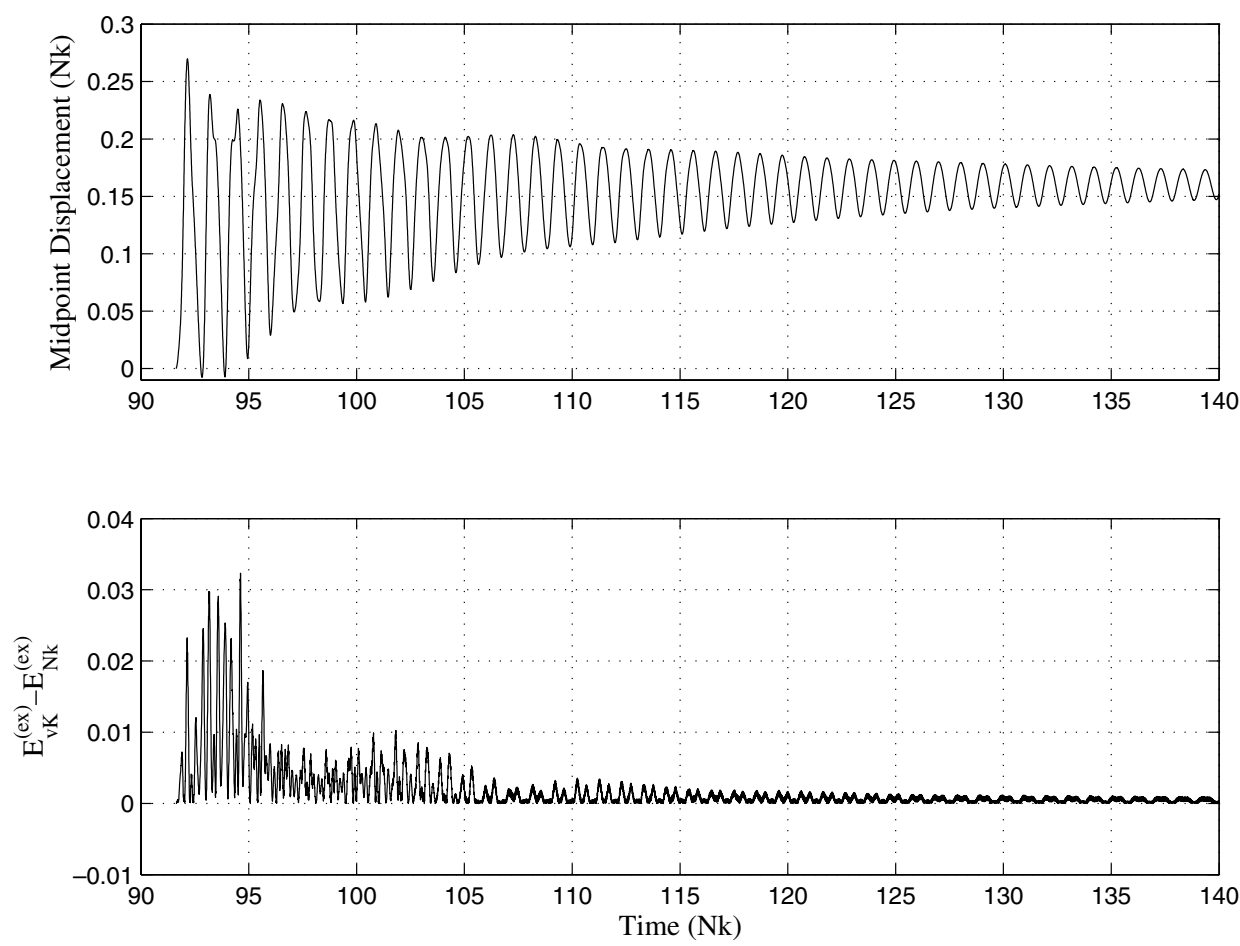

Fig. 13. Constrained $L^{2}$ projection of pressure to a time dependent quadratic and $L^{2}$ projection of velocity. The midpoint displacement (top) and difference in external forces energy over the wetted surface $\Delta_{\text {wet }} E^{\mathrm{ex}}$ (bottom). 


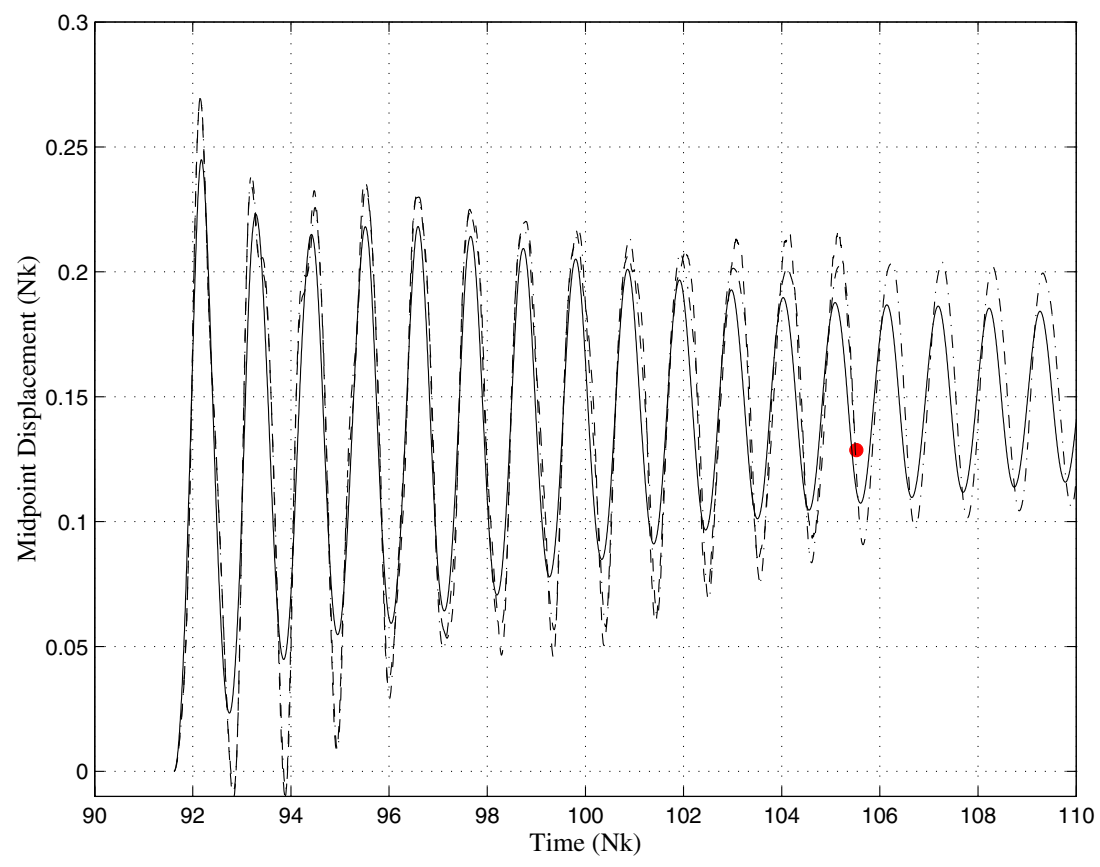

Fig. 14. The midpoint displacement as presented in Fig. 10 (solid and dashed) compared to Case 2.B: constrained $L^{2}$ projection of pressure to a time dependent quadratic and $L^{2}$ projection of velocity (dash-dot).

3rd-order on the von-Kármán side (VP-3), there was a noticeable difference in the midpoint displacement when compared to using $L^{2}$ projections between the two spaces directly below $t_{\mathrm{F}}=100$. Only after the Case 1.C solution starts to blow up do you see (expected) discrepancies between Case 1.C and Case 2.C. In Fig. 14 we present for comparison the midpoint displacement obtained when the force is projected to a time dependent quadratic function (PP-3), and velocity is projected using $L^{2}$ projections (VP-2). We observe that the discrepancy between the use of $L^{2}$ and smoothed $L^{2}$ midpoint displacements is drastically reduced.

Further tests not expounded upon here were accomplished with the fluid discretization increased to seventh-order polynomials and tests with the mass ratio decreased to $M R=50$. In all cases examined, projecting the force to a time-dependent quadratic function was sufficient to maintain stability.

Remark 8. The non-linear terms in Navier-Stokes are often treated in a collocation manner (as in $\mathscr{N} \varepsilon \kappa \mathscr{T} \alpha r$ ). Marginal resolution can result in aliasing - which can cause instability [27]. This problem can in general be overcome by: (i) over-integration [42] or (ii) by adding filtering or other dissipative mechanics (like in loworder methods). Note that these facts are stated without regard for the FSI system. A fluid solver with no moving boundaries may suffer from instability if marginal resolution is used (i.e. the solution used is not sufficient to capture the phenomena). In the case of this work, wiggles were introduced at the structural interface due to our choice of projections (in particular, we were initially only concerned with global conservation and not local features). To verify that aliasing issues (excited and exacerbated by choosing projection methods which do not respect monotonicity), we ran both over-integrated runs and finite volume runs. The over-integration delayed the onset of the instability as predicted in [27], but did not eliminate it as there are rational terms in the compressible Navier-Stokes equations; the finite volume discretization of the fluid eliminates the instability at the price of resolution.

\section{Summary and future work}

High-order finite element methods combine the meshing flexibility of standard finite element methods with the resolution fidelity of global high-order (spectral) methods, and have been shown to be a valuable tool in solving engineering problems in fluid mechanics and solid mechanics. As FSI problems require increased res- 
olution needs in both the fluid and the structural simulations, high-order methods are a natural choice for simulating either the fluid, the structure or both. It should be noted, however, that the increased meshing flexibility and resolution fidelity have as consequences decreased numerical dissipation and relaxation of monotonicity requirements. For one to exploit the benefits of high-order methods for fluid-structure interaction, it is imperative that one understand and respect the influences of decreased numerical dissipation and relaxed monotonicity on their proposed coupling schemes.

In this work, we have provided a case study which illustrates how to, and how not to, couple high-order methods for fluid-structure interaction problems. We started by naively coupling two high-order codes together using projection schemes commonly used in low-order methods. Although we obtained computational results for short-time, we observed that the simulations were not stable for long-time integration. As is normally done in low-order methods, we then migrated to projection schemes which were conservative (in our case, conserving total force and total momentum over the wetted surface) in hopes that stability would follow (and in the hopes of more accurately capturing what must hold in the physics). However, conservation was not sufficient. It was only when we addressed first-principles - that is, we examined the space of functions from which we were projecting and to which we were projecting - did it become apparent that properties tacitly assumed true in low-order methods did not hold when using high-order methods. Although one could argue that global conservation properties are crucial for capturing the physics of the problem, their satisfaction appears subordinate to maintaining proper trace-space relations. It was only when conservative projections which respected the properties of the underlying discretization function spaces were used did we obtain stable and accurate solutions.

In conclusion, this work demonstrates that coupling high-order finite element schemes requires one to choose projection algorithms which are conservative, preserve the physical features of interest and respect the mathematical properties of the underlying discretizations. This means that conservative projection schemes may need to be modified (such as using general Sobolev norm projections [11]) or augmented (such as with filtering, spectral vanishing viscosity, etc.) in order for the full benefit of the high-order method coupling to be realized.

Although the pseudo-spectral method was used for the discretization of the structure, the FSI ingredients for a stable and accurate weakly-coupled system carry over to high-order finite element methods. In the future, we would continue to ferret out the nuances of temporal and spatial coupling of high-order discretizations in FSI. With respect to temporal coupling, we are interested in exploring a wider range of mass and rigidity ratios, especially for ocean FSI problems, and blood flow in arteries. With respect to spatial coupling, we are interested in investigating what further modifications to conservative projections are necessary for the coupling of two arbitrary high-order discretizations used for solving FSI problems.

\section{Acknowledgment}

The first author gratefully acknowledges the support provided under NSF Career Award (Kirby) NSFCCF0347791 and the computational support provided by the Scientific Computing and Imaging Institute at the University of Utah (specifically the NCRR Center for Bioelectric Field Modeling, Simulation, and Visualization - www.sci.utah.edu/ncrr) upon which simulations for this paper were accomplished.

The second and third authors gratefully acknowledge the support of this work by the Office of Naval Research (Ocean Engineering and Marine Systems) and the United States-Israel Binational Science Foundation.

\section{Appendix A. Governing equations for the fluid}

The fluid is represented by the non-dimensionalized three-dimensional compressible Navier-Stokes equations:

$$
\dot{\boldsymbol{W}}+\nabla \cdot \mathbf{F}\left(\boldsymbol{W}, \boldsymbol{U}^{g}\right)=\frac{1}{R e_{\infty}} \nabla \cdot \mathbf{F}^{v}(\boldsymbol{W}) \quad \text { on } \Omega_{\mathrm{F}}(t)
$$

where the vector $\boldsymbol{W}^{\mathrm{T}}(\boldsymbol{x}, t)=\left(W_{1}, W_{1} W_{2}, W_{1} W_{3}, W_{1} W_{4}, W_{5}\right)^{\mathrm{T}}, \boldsymbol{x} \in \Omega_{\mathrm{F}}(t)$ denotes the fluid solution variables of density, momentum in the three coordinate directions with $\left(W_{2}, W_{3}, W_{4}\right)^{\mathrm{T}}$ denoting the local fluid velocity, and 
total energy, respectively, the vector $\boldsymbol{U}^{g}$ denotes the velocity of the moving frame within the ALE formulation, $R e_{\infty}$ denotes the Reynolds number, and $\mathbf{F}$ and $\mathbf{F}^{v}$ denote that inviscid and viscous fluxes respectively.

The physical quantities are denoted by an asterisk, thus the fluid density is $W_{1}^{*}$, the pressure is $p^{*}$, the spatial coordinates are $x_{i}^{*}$, the three velocity components are $W_{2}^{*}, W_{3}^{*}, W_{4}^{*}$, and the plate's length is $a^{*}$ (characteristic length). The non-dimensional quantities with respect to the physical values are:

$$
\begin{aligned}
& W_{2}=\frac{W_{2}^{*}}{v_{\infty}^{*}}, \quad W_{3}=\frac{W_{3}^{*}}{v_{\infty}^{*}}, \quad W_{4}=\frac{W_{4}^{*}}{v_{\infty}^{*}}, \quad x_{1}=\frac{x_{1}^{*}}{\left(a^{*} / 2\right)}, \quad x_{2}=\frac{x_{2}^{*}}{\left(a^{*} / 2\right)}, \quad t=t^{*} \frac{v_{\infty}^{*}}{\left(a^{*} / 2\right)} \\
& p=\frac{p^{*}}{p_{\infty}^{*}}=\frac{p^{*}}{\left(W_{1}\right)_{\infty}^{*}\left(v_{\infty}^{*}\right)^{2}}, \quad \text { where } v_{\infty}^{*}=\sqrt{\frac{p_{\infty}^{*}}{\left(W_{1}\right)_{\infty}^{*}} .}
\end{aligned}
$$

where the dynamic viscosity is $\mu_{\infty}^{*}$ and thermal conductivity $\kappa_{\infty}^{*}$.

The connection between the pressure $p$ and the solution vector can be obtained via the perfect gas law:

$$
p=\frac{W_{1} \tau}{\gamma M a_{\infty}^{2}}
$$

where $\tau=\frac{\tau^{*}}{\tau_{\infty}}$ is the non-dimensional temperature (computed from the energy $W_{5}$ ), and $M a_{\infty}$ is the Mach number.

With the above notation the compressible Navier-Stokes can be written as follows:

$$
\begin{aligned}
& \frac{\partial \boldsymbol{W}}{\partial t}+\frac{\partial}{\partial x_{1}}\left(\begin{array}{c}
W_{1} W_{2} \\
W_{1} W_{2}^{2}+p \\
W_{1} W_{2} W_{3} \\
W_{1} W_{2} W_{4} \\
\left(W_{5}+p\right) W_{2}
\end{array}\right)+\frac{\partial}{\partial x_{2}}\left(\begin{array}{c}
W_{1} W_{3} \\
W_{1} W_{3} W_{2} \\
W_{1} W_{3}^{2}+p \\
W_{1} W_{3} W_{4} \\
\left(W_{5}+p\right) W_{3}
\end{array}\right)+\frac{\partial}{\partial x_{3}}\left(\begin{array}{c}
W_{1} W_{4} \\
W_{1} W_{4} W_{2} \\
W_{1} W_{4} W_{3} \\
W_{1} W_{4}^{2}+p \\
\left(W_{5}+p\right) W_{4}
\end{array}\right) \\
& =\frac{\mu}{R e_{\infty}}\left\{\frac{\partial}{\partial x_{1}}\left(\begin{array}{c}
0 \\
\frac{2}{3}\left(2 \frac{\partial W_{2}}{\partial x_{1}}-\frac{\partial W_{3}}{\partial x_{2}}-\frac{\partial W_{4}}{\partial x_{3}}\right) \\
\left(\frac{\partial W_{2}}{\partial x_{2}}+\partial W_{3} \partial x_{1}\right) \\
\left(\frac{\partial W_{2}}{\partial x_{3}}+\frac{\partial W_{4}}{\partial x_{1}}\right) \\
\frac{2}{3} W_{2}\left(2 \frac{\partial W_{2}}{\partial x_{1}}-\frac{\partial W_{3}}{\partial x_{2}}-\frac{\partial W_{4}}{\partial x_{3}}\right)+W_{3}\left(\frac{\partial W_{2}}{\partial x_{2}}+\frac{\partial W_{3}}{\partial x_{1}}\right)+W_{4}\left(\frac{\partial W_{2}}{\partial x_{3}}+\frac{\partial W_{4}}{\partial x_{1}}\right)+\kappa \frac{\gamma}{\mu P r_{\infty}} \frac{\partial \tau}{\partial x_{1}}
\end{array}\right)\right. \\
& +\frac{\partial}{\partial x_{2}}\left(\begin{array}{c}
0 \\
\left(\frac{\partial W_{2}}{\partial x_{2}}+\frac{\partial W_{3}}{\partial x_{1}}\right) \\
\frac{2}{3}\left(2 \frac{\partial W_{3}}{\partial x_{2}}-\frac{\partial W_{2}}{\partial x_{1}}-\frac{\partial W_{3}}{\partial x_{3}}\right) \\
\mu\left(\frac{\partial W_{2}}{\partial x_{3}}+\frac{\partial W_{4}}{\partial x_{2}}\right) \\
W_{2}\left(\frac{\partial W_{2}}{\partial x_{2}}+\partial W_{3} \partial x_{1}\right)+\frac{2}{3} W_{3}\left(2 \frac{\partial W_{3}}{\partial x_{2}}-\frac{\partial W_{2}}{\partial x_{1}}-\frac{\partial W_{4}}{\partial x_{3}}\right)+W_{4}\left(\frac{\partial W_{3}}{\partial x_{3}}+\partial W_{4} \partial x_{2}\right)+\kappa \gamma \mu P r_{\infty} \frac{\partial \tau}{\partial x_{2}}
\end{array}\right) \\
& \left.+\frac{\partial}{\partial x_{3}}\left(\begin{array}{c}
0 \\
\left(\frac{\partial W_{2}}{\partial x_{3}}+\frac{\partial W_{4}}{\partial x_{1}}\right) \\
\left(\frac{\partial W_{3}}{\partial x_{3}}+\frac{\partial W_{4}}{\partial x_{2}}\right) \\
\frac{2}{3}\left(2 \frac{\partial W_{4}}{\partial x_{3}}-\frac{\partial W_{2}}{\partial x_{1}}-\frac{\partial W_{3}}{\partial x_{2}}\right) \\
W_{2}\left(\frac{\partial W_{2}}{\partial x_{3}}+\frac{\partial W_{4}}{\partial x_{1}}\right)+W_{2}\left(\frac{\partial W_{3}}{\partial x_{3}}+\frac{\partial W_{4}}{\partial x_{2}}\right)+W_{4} \frac{2}{3}\left(2 \frac{\partial W_{4}}{\partial x_{3}}-\frac{\partial W_{2}}{\partial x_{1}}-\frac{\partial W_{3}}{\partial x_{2}}\right)+\kappa \frac{\gamma}{\mu P r_{\infty}} \frac{\partial \tau}{\partial x_{3}}
\end{array}\right)\right\}
\end{aligned}
$$

The temperature $\tau$ in (27) can be expressed using (26) in terms of $p, W_{1}, M a_{\infty}$. 
To accommodate moving grids, we use the arbitrary-Lagrangian-Eulerian (ALE) formulation as presented in [10]. Here we briefly review the ALE formulation. We need not give special consideration to the viscous terms because the viscous contributions do not depend on the grid velocity $\mathbf{U}^{g}$.

Using the Reynolds transport theorem we can write the Euler equations (i.e. $\operatorname{set} \mathbf{F}^{v}(\boldsymbol{W})=0$ in (1)) in the ALE framework following the formulation proposed in [43] as

$$
\dot{\boldsymbol{W}}+\nabla \cdot \mathbf{H}=-\left(\nabla \cdot \boldsymbol{U}^{g}\right) \boldsymbol{W}
$$

where the ALE flux term $\mathbf{H}=\left(H_{1}, H_{2}, H_{3}\right)$ is defined as a row vector containing three column vectors (denoted by the subscript $i$ ) given by:

$$
H_{i}=\left(W_{i+1}-U_{i}^{g}\right) \boldsymbol{W}+p\left[0, \delta_{1 i}, \delta_{2 i}, \delta_{3 i}, W_{i+1}\right]^{t}, \quad i=1,2,3 .
$$

where $W_{i+1}$ is the $(i+1)$ th component of the velocity vector and $U_{i}^{g}$ is the $i$ th component of the grid velocity vector $\mathbf{U}^{g}$. Observe that we can recover the Euler flux $\mathbf{F}$ by setting $\mathbf{U}^{g}=0$. Also observe that we can re-write $\mathbf{H}$ in terms of $\mathbf{F}$ as follows: $H_{i}=F_{i}-U_{i}^{g} \boldsymbol{W}, i=1,2,3$. Now if we write the ALE Euler equations in terms of the Euler flux then the source term on the right-hand-side of Eq. (28) is eliminated, and we obtain:

$$
\dot{W}+\nabla \cdot \mathbf{F}-\left(\mathbf{U}^{g} \cdot \nabla\right) \boldsymbol{W}=0,
$$

which can then be recast in the standard quasi-linear form (written in matrix notation) as:

$$
\dot{\boldsymbol{W}}+\left[A_{i}-U_{i}^{g} \mathbf{I}\right] \boldsymbol{W}_{, i}=0,
$$

where $A_{i}=\partial F_{i} / \partial \boldsymbol{W}(i=1,2,3)$ is the flux Jacobian and $\mathbf{I}$ is the unit matrix. In this form it is straightforward to obtain the corresponding characteristic variables since the ALE Jacobian matrix can be written

$$
A_{i}^{\mathrm{ALE}} \equiv\left[A_{i}-U_{i}^{g} \mathbf{I}\right]=R_{i} \cdot\left[\Lambda_{i}-U_{i}^{g} \mathbf{I}\right] \cdot L_{i},
$$

where brackets denote matrix. Here the matrix $\Lambda$ contains on the diagonal the eigenvalues of the original Euler Jacobian matrix $A$, and $R$ and $L$ are the right- and left-eigenvector matrices, respectively, containing the corresponding eigenvectors of $A$. Note that the shifted eigenvalues of the ALE Jacobian matrix do not change the corresponding eigenvectors in the characteristic decomposition.

\section{Appendix B. Governing equations for the structure}

We consider a square plate made of an isotropic elastic material of dimensions $a^{*} \times a^{*} \times h^{*}$, having one of its dimensions $h^{*}$ much smaller compared to the other two, i.e. $h^{*} \ll a^{*}$. Let us denote the mid-plane surface by $\Omega_{\mathrm{S}}^{*}=\left[0, a^{*}\right] \times\left[0, a^{*}\right] \in \mathbb{R}^{2}$, see Fig. 1 .

Let $E^{*}, v, c^{*}, \rho_{\mathrm{S}}^{*}$ denote the Young modulus, Poisson ratio, structural viscosity coefficient and density of the plate's material. We assume no body forces are applied on the plate, and on its upper surface, traction loading $g_{i}^{* \pm \frac{k^{*}}{2}}$ are applied. These are of course prescribed functions of $x_{1}^{*}, x_{2}^{*}$ and $t^{*}$ alone.

Let $\boldsymbol{u}^{*}\left(\boldsymbol{x}^{*}, t^{*}\right)=\left(u^{*} v^{*} w^{*}\right)^{\mathrm{T}}$ denote the mid-plane displacement vector (the deflection of the mid-surface of the plate is $\left.w^{*}\right)$. The complete von-Kármán set of equations in terms of the three displacement functions $\boldsymbol{u}^{*}$ is given on $\Omega_{\mathrm{S}}^{*} \in \mathbb{R}^{2}$ in [32].

By performing the following change of variables:

$$
\begin{aligned}
& u=\frac{\left(a^{*} / 2\right) u^{*}}{\left(h^{*}\right)^{2}}, \quad v=\frac{\left(a^{*} / 2\right) v^{*}}{\left(h^{*}\right)^{2}}, \quad w=\frac{w^{*}}{\left(h^{*}\right)}, \quad x_{1}=\frac{x_{1}^{*}}{\left(a^{*} / 2\right)}, \quad x_{2}=\frac{x_{2}^{*}}{\left(a^{*} / 2\right)}, \\
& t=t^{*} \sqrt{\frac{D^{*}}{\rho_{\mathrm{S}}^{*} h^{*}\left(a^{*} / 2\right)^{4}}} \quad g=\frac{g^{*}\left(a^{*} / 2\right)^{4}}{D^{*} h^{*}}, \quad h=\frac{h^{*}}{\left(a^{*} / 2\right)}, \quad c=c^{*} \sqrt{\frac{\left(a^{*} / 2\right)^{4} h^{*}}{\rho_{\mathrm{S}}^{*} D^{*}}}
\end{aligned}
$$

where $D^{*} \stackrel{\text { def }}{=} \frac{E^{*}\left(h^{*}\right)^{3}}{12\left(1-v^{2}\right)}$ is the flexural rigidity, the non-dimensional von-Kármán system over a quadrilateral domain $\Omega=[0,2] \times[0,2]$ is given by the following three coupled equations: 


$$
\begin{aligned}
& w_{, t t}+c w_{, t}-\frac{h^{2}}{12} \Delta w_{, t t}+\Delta^{2} w-12\left[\left(u_{, 1}+\frac{1}{2} w_{, 1}^{2}\right)\left(w_{, 11}+v w_{, 22}\right)+\left(v_{, 2}+\frac{1}{2} w_{, 2}^{2}\right)\left(w_{, 22}+v w_{, 11}\right)\right. \\
& \left.\quad+(1-v)\left(v_{, 1}+u_{, 2}+w_{, 1} w_{, 2}\right) w_{, 12}\right]=g_{3} \\
& \frac{h^{2}}{6}\left(u_{, t t}+c u_{, t}\right) \\
& \quad-\left[2 u_{, 11}+(1+v) v_{, 12}+(1-v) u_{, 22}+2 w_{, 1} w_{, 11}+(1+v) w_{, 2} w_{, 12}+(1-v) w_{, 1} w_{, 22}\right]=g_{2} \\
& \frac{h^{2}}{6}\left(v_{, t t}+c v_{, t}\right) \\
& \quad-\left[2 v_{, 22}+(1+v) u_{, 12}+(1-v) v_{, 11}+2 w_{, 2} w_{, 22}+(1+v) w_{, 1} w_{, 12}+(1-v) w_{, 2} w_{, 11}\right]=g_{3}
\end{aligned}
$$

where $\bullet, i=\frac{\partial \bullet}{\partial x_{i}}$, and $\Delta$ is the Laplace operator in the plane $x_{1}, x_{2}$.

The system is accompanied by Dirichlet boundary conditions (denoted by "hard clamped") which are:

$$
u=v=w=\partial_{n} w=0 \quad \text { on } \partial \Omega_{\mathrm{S}}
$$

The third term in (32) and the first two time-dependent terms in (33) and (34) are of order $h^{2}$ and are commonly neglected. Thus, numerical treatment of the simplified system is well behaved and simple, and as shown in [44], accurate for $h^{*} / a^{*}$ ratios smaller than $1 / 20$, hence we use herein the simplified version of the von-Kármán system, i.e. neglecting the two terms that are multiplied by $h^{2}$ in (33) and (34).

\section{Appendix C. Unified non-dimensionalization between the fluid and the structure}

To differentiate between the non-dimensional quantities as used within the fluid solver versus the quantities within the structural solver, we will use the subscript $S$ to refer to the quantities in the von-Kármán structural solver and $F$ to refer to these in the $\mathscr{N} \varepsilon \kappa \mathscr{T} \alpha r$ fluid solver.

We define the following rigidity and mass ratios, respectively:

$$
R R=\frac{E^{*}}{p_{\infty}^{*}}, \quad M R=\frac{\rho_{\mathrm{S}}^{*}}{\rho_{\infty}^{*}},
$$

where the definitions of these characteristic variables were given in Section 2.1.

Comparing the non-dimensionalizations given in Appendix A for the fluid equations with these in Appendix B for the structural equations, we may derive conversion factors for equating fields derived from the fluid variables and fields derived from the structural variables. In Table 3 we present the conversion factors required in this work.

Consider, for example, an Aluminum 2024 plate with a thickness to half-length ratio (see Fig. 1) of $h=0.1$, $v=0.3, E^{*}=73.1 \mathrm{GPa}=73.1 \times 10^{9} \mathrm{~N} / \mathrm{m}^{2}$, and $\rho_{\mathrm{S}}^{*}=2700 \mathrm{~kg} / \mathrm{m}^{3}$, in a flow where $p_{\infty}^{*}=101300 \mathrm{~N} / \mathrm{m}^{2}$, and $\rho_{\infty}^{*}=1.229 \mathrm{~kg} / \mathrm{m}^{3}$ are taken as the standard atmospheric air pressure and density, then:

Table 3

Conversion factors for transferring information from fields based upon the fluid non-dimensionalization to fields based upon the structural non-dimensionalization

\begin{tabular}{lr}
\hline Time & $t_{\mathrm{S}}=t_{\mathrm{F}} \sqrt{\frac{h^{2}}{C_{1}}}$ \\
Pressure (load) & $g_{\mathrm{S}}=p_{\mathrm{F}} \frac{C_{1}}{h^{4} M R}$ \\
Displacements & $u_{\mathrm{S}}=u_{\mathrm{F}} \sqrt{\frac{C_{1}}{h^{6}}}$ \\
& $v_{\mathrm{S}}=v_{\mathrm{F}} \sqrt{\frac{C_{1}}{h^{6}}}$ \\
$w_{\mathrm{S}}=w_{\mathrm{F}} \sqrt{\frac{C_{1}}{h^{4}}}$ & $\dot{w}_{\mathrm{S}}=\dot{w}_{\mathrm{F}} \sqrt{\frac{C_{1}}{h^{4}}}$ \\
Velocity (normal to surface) & \\
\hline
\end{tabular}

$C_{1} \stackrel{\text { def }}{=} \frac{12\left(1-v^{2}\right) M R}{R R}$ and $h=h^{*} /\left(a^{*} / 2\right)$. 


$$
R R=721,618.95, \quad M R=2196.908, \quad \Rightarrow \frac{M R}{R R}=0.00304 .
$$

By Table 3 one finds that:

$$
t_{\mathrm{S}}=0.5488 t_{\mathrm{F}}
$$

or, another way to look at it is the following. An interval of $1 \mathrm{~s}$ is equivalent to

$$
t_{\mathrm{F}}=1 \sqrt{\frac{p_{\infty}^{*}}{\rho_{\infty}^{*}}} \frac{1}{a^{*}}=1 \sqrt{\frac{101300}{1.229}}=287.097 \mathscr{N} \varepsilon \kappa \mathscr{T} \alpha r \text { time units, }
$$

and using (38), it is also equivalent to $t_{\mathrm{S}}=0.5488 \times 287.097=157.558$ von-Kármán time units. Thus, if we chose a time-step $\Delta t_{\mathrm{F}}=10^{-5}$, this corresponds to physical time $\Delta t=10^{-5} / 287.097=3.483 \times 10^{-8} \mathrm{~s}$, and the time-step in the von-Kármán solver so it receives and transfers data at the same physical time should be: $\Delta t_{\mathrm{vk}}=3.483 \times 10^{-8} \times 157.558=5.48798 \times 10^{-6}$.

\section{References}

[1] R.E. Gordnier, M.R. Visbal, Development of a three-dimensional viscous aeroelastic solver for nonlinear panel flutter, J. Fluids Struct. 16 (4) (2002) 497-527.

[2] C. Farhat, M. Lesoinne, Two efficient staggered algorithms for the serial and parallel solution of three-dimensional nonlinear transient aeroelastic problems, Comput. Meth. Appl. Mech. Engrg. 182 (2000) 499-515.

[3] E.F. Sheta, S.G. Rock, L.J. Huttsell, Characteristics of vertical tail buffet of F/A-18 aircraft, AIAA-2001-0710, 2001.

[4] C. Farhat, P. Geuzaine, G. Brown, Formulation to the prediction of the aeroelastic parameters of an F-16 fighter, Comput. Fluids 32 (1) (2003) 3-29.

[5] S. Sirisup, G.E. Karniadakis, Y. Yang, D. Rockwell, Wave-structure interaction: simulation driven by quantitative imaging, Proc. Roy. Soc. 460 (2042) (2004) 729-755.

[6] H.F. Younis, M.R. Kaazempur-Mofrad, R.C. Chan, A.G. Isasi, D.P. Hinton, A.H. Chau, L.A. Kim, R.D. Kamm, Hemodynamics and wall mechanics in human carotid bifurcation and its consequences for atherogenesis: investigation of inter-individual variation, Biomechan. Model Mechanobiol. 3 (2004) 1732.

[7] B.A. Szabó, I. Babuška, Finite Element Analysis, Wiley, New York, 1991.

[8] B. Szabó, A. Düster, E. Rank, The p-version of the finite element method, in: E. Stein, R. de Borst, T.J.R. Hughes (Eds.), Encyclopedia of Computational Mechanics, Wiley, 2004, pp. 119-139 (Chapter 5).

[9] G.E. Karniadakis, S.J. Sherwin, Spectral/hp Element Methods for CFD, second ed., Oxford University Press, UK, 2005.

[10] I. Lomtev, R.M. Kirby, G.E. Karniadakis, A discontinuous Galerkin ALE method for compressible viscous flows in moving domains, J. Comput. Phys. 155 (1999) 128-159.

[11] X. Jiao, M.T. Heath, Common-refinement based data transfer between nonmatching meshes in multiphysics simulations, Intt. J. Numer. Meth. Engrg. 61 (14) (2004) 2402-2427.

[12] O.O. Bendiksen, A new approach to computational aeroelasticity, AIAA Paper, 91-0939-CP, AIAA, 1991.

[13] E.H. van Brummelen, S.J. Hulshoff, R. de Borst, Energy conservation under incompatibility for fluid structure interaction problems, Comput. Meth. Appl. Mech. Engrg. 192 (25) (2003) 2727-2748.

[14] C. Michler, E.H. van Brummelen, S.J. Hulshoff, R. de Borst, The relevance of conservation for stability and accuracy of numerical methods for fluid structure interaction, Comput. Meth. Appl. Mech. Engrg. 192 (37-38) (2003) 4195-4215.

[15] S. Piperno, C. Farhat, Design of efficient partitioned procedures for the transient solution of aeroelastic problems, Revue Europeanne des Elements Finis: Fluid-Structure Interaction 9 (6-7) (2000) 655-680.

[16] E.H. Dowell, K.C. Hall, Modeling of fluid-structure interaction, Ann. Rev. Fluid Mech. 33 (2001) 445-490.

[17] B. Cockburn, G.E. Karniadakis, C-W. Shu, Discontinuous Galerkin Methods: Theory, Computation and Applications, Springer, 2000.

[18] A. Düster, S. Hartmann, E. Rank, p-Fem applied to finite isotropic hyperelastic bodies, Comput. Meth. Appl. Mech. Engrg. 192 (2003) 5147-5166.

[19] S.A. Morton, R.B. Melville, M.R. Visbal, Accuracy and coupling issues of aeroelastic Navier-Stokes solutions on deforming meshes, J. Aircraft 35 (5) (1998) 798-805.

[20] D.P. Rizzeta, M.R. Visbal, Comparative numerical study of two turbulence models for airfoil static and dynamic stall, AIAA J. 31 (4) (1993) 784-786.

[21] S. Piperno, C. Farhat, B. Larrouturou, Partitioned procedures for the transient solution of coupled aeroelastic problems: Part I: Model problem, theory, and two-dimensional application, Comput. Meth. Appl. Mech. Engrg. (1995) 79-112.

[22] C. Farhat, M. Lesoinne, P. LeTallec, Load and motion transfer algorithms for fluid/structure interaction problems with nonmatching discrete interfaces: momentum and energy conservation, optimal discretization and application to aeroelasticity, Comput. Meth. Appl. Mech. Engrg. 157 (1998) 95-114. 
[23] S. Piperno, C. Farhat, Partitioned procedures for the transient solution of coupled aeroelastic problems: Part II: Energy transfer analysis and three-dimensional applications, Comput. Meth. Appl. Mech. Engrg. 190 (24) (2001) 3147-3170.

[24] C. Farhat, K.G. van der Zee, P. Geuzaine, Provably second-order time-accurate loosely-coupled solution algorithms for transient nonlinear computational aeroelasticity, Comput. Meth. Appl. Mech. Engrg. 195 (17-18) (2006) 1973-2001.

[25] R.M. Kirby, Z. Yosibash, Solution of von-Kármán dynamic non-linear plate equations using a pseudo-spectral method, Comput. Meth. Appl. Mech. Engrg. 193 (6-8) (2004) 575-599.

[26] W.S. Don, D. Gottlieb, Spectral simulation of unsteady flow past a cylinder, Comput. Meth. Appl. Mech. Engrg. 80 (1990) 39-58.

[27] R.M. Kirby, G.E. Karniadakis, Under-resolution and diagnostics in spectral simulations of complex-geometry flows, in: D. Drikakis, B. Geurts (Eds.), Turbulent Flow Computation, Kluwer Academic Publishers., The Netherlands, 2001, pp. 1-42.

[28] R.M. Kirby, T.C. Warburton, S.J. Sherwin, A. Beskok, G.E. Karniadakis, The $\mathscr{N} \varepsilon \kappa \mathscr{T} \alpha r$ code: Dynamic simulations without remeshing, in: Proceedings of the 2nd International Symposium on Computational Technologies for Fluid/Thermal/Chemical Systems with Industrial Applications, vol. 397, ASME PVP Division, 1999.

[29] M. Lesoinne, C. Farhat, Geometric conservation laws for flow problems with moving boundaries and deformable meshes, and their impact on aeroelastic computations, Comput. Meth. Appl. Mech. Engrg. 134 (1996) 71-90.

[30] C. Farhat, P. Geuzaine, C. Grandmont, The discrete geometric conservation law and the nonlinear stability of ALE schmemes for the solution of flow problems on moving grids, J. Comput. Phys. 174 (2001) 669-694.

[31] H. Guillard, C. Farhat, On the significance of the geometric conservation law for flow computations on moving meshes, Comput. Meth. Appl. Mech. Engrg. 190 (2000) 1467-1482.

[32] Z. Yosibash, R.M. Kirby, D. Gottlieb, Pseudo-spectral methods for the solution of the von-Kármán dynamic non-linear plate system, J. Comput. Phys. (2004).

[33] W. Hundsdorfer, J.G. Verwer, Numerical Solution of Time-Dependent Advection-Diffusion-Reaction EquationsSpringer Series in Computational Mathematics, Springer, New York, 2003.

[34] J.R. Cebral, R. Löhner, Conservative load projection and tracking for fluid-structure problems, AIAA J. 35 (4) (1997) $687-692$.

[35] Q. Zhang, T. Hisada, Studies of the strong coupling and weak coupling methods in FSI analysis, Int. J. Numer. Meth. Engrg. 60 (2004) 2013-2029.

[36] G.A. Holzapfel, Nonlinear Solid Mechanics, Wiley, New York, 2000.

[37] R.L. Panton, Incompressible Flows, Wiley, New York, 1996.

[38] Y. Maday, C. Mavriplis, A.T. Patera, Non-conforming mortar element methods: applications to spectral discretizations, in: Domain Decompostion Methods, SIAM, Philadelphia, 1989.

[39] G. Anagnostou, Y. Maday, C. Mavriplis, A.T. Patera, On the mortar element method: generalization and implementation, in: R. Glowinski (Ed.), Third International Conference on Domain Decomposition Methods for Partial Differential Equations, SIAM, Philadelphia, 1990.

[40] C. Bernardi, Y. Maday, A.T. Patera, A new nonconforming approach to domain decomposition: the mortar element method, in: H Brezis, J.L. Lions (Eds.), Nonlinear Partial Differential Equations and their Applications, Pitman/Wiley, London/New York, 1992.

[41] C. Canuto, M.Y. Hussaini, A. Quarteroni, T.A. Zang, Spectral Methods in Fluid Mechanics, Springer, New York, 1987.

[42] R.M. Kirby, G.E. Karniadakis, De-aliasing on non-uniform grids: algorithms and applications, J. Comp. Phys. 191 (2003) $249-264$

[43] C.S. Venkatasubban, A new finite element formulation for ALE Arbitrary Lagrangian Eulerian compressible fluid mechanics, Int. J. Engrg. Sci. 33 (12) (1995) 1743-1762.

[44] Z. Yosibash, R.M. Kirby, Dynamic response of various von-Karman non-linear plate models and their 3-d counterparts, Int. J. Solids Struct. 42 (9-10) (2005) 2517-2531. 